Provided for non-commercial research and education use. Not for reproduction, distribution or commercial use.

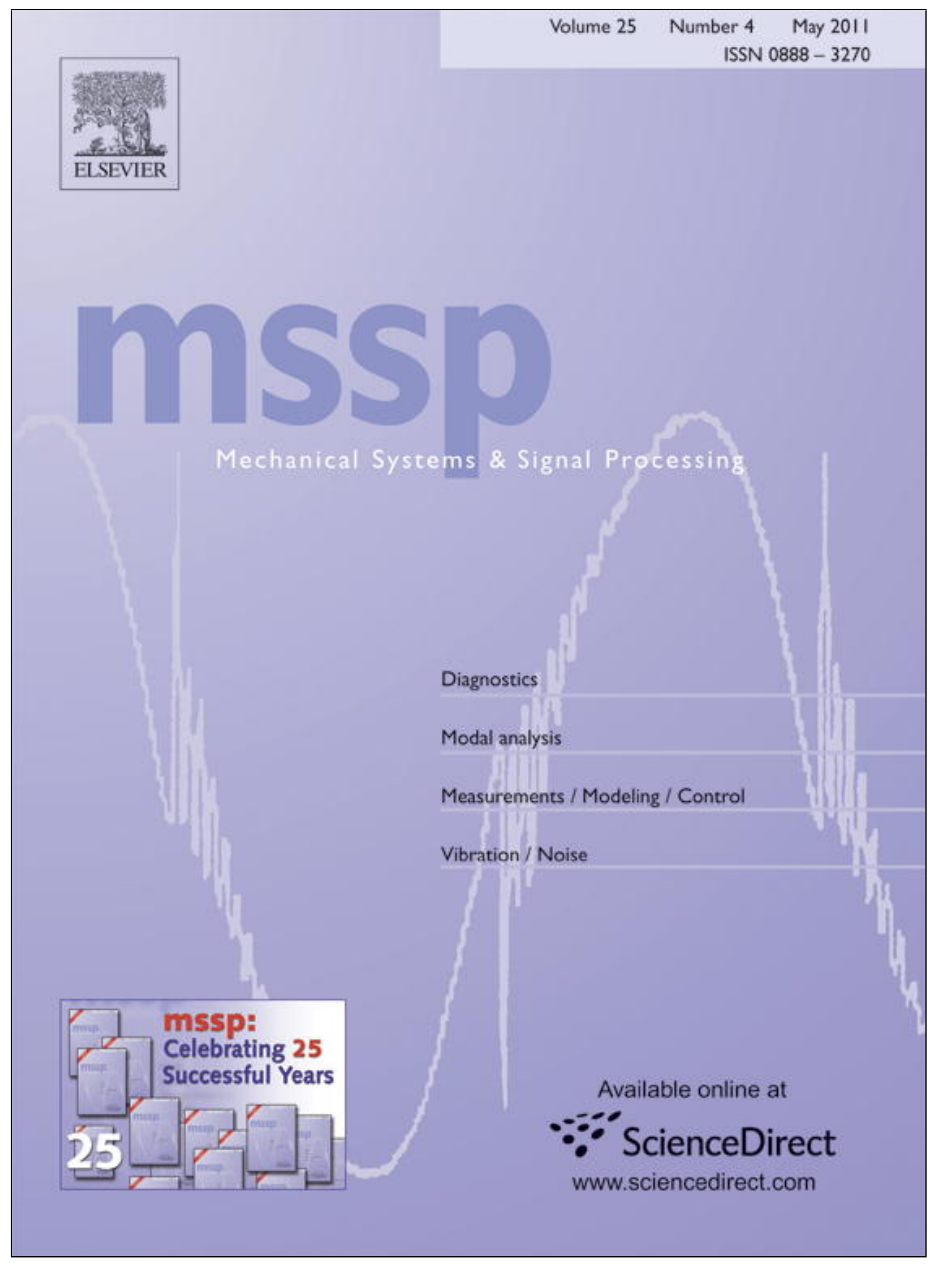

This article appeared in a journal published by Elsevier. The attached copy is furnished to the author for internal non-commercial research and education use, including for instruction at the authors institution and sharing with colleagues.

Other uses, including reproduction and distribution, or selling or licensing copies, or posting to personal, institutional or third party websites are prohibited.

In most cases authors are permitted to post their version of the article (e.g. in Word or Tex form) to their personal website or institutional repository. Authors requiring further information regarding Elsevier's archiving and manuscript policies are encouraged to visit:

http://www.elsevier.com/copyright 


\title{
OPAX: A new transfer path analysis method based on parametric load models
}

\author{
Karl Janssens ${ }^{\mathrm{a}, *}$, Peter Gajdatsy ${ }^{\mathrm{b}}$, Ludo Gielen ${ }^{\mathrm{a}}$, Peter Mas ${ }^{\mathrm{a}}$, Laurent Britte ${ }^{\mathrm{a}}$, \\ Wim Desmet ${ }^{\mathrm{b}}$, Herman Van der Auweraer ${ }^{\mathrm{a}}$ \\ a LMS International NV, Interleuvenlaan 68, B-3001 Leuven, Belgium \\ ${ }^{\mathrm{b}}$ K.U. Leuven, Department of Mechanical Engineering, Celestijnenlaan 300B, 3001 Leuven, Belgium
}

\section{A R T I C L E I N F O}

\section{Article history:}

Received 25 May 2010

Received in revised form

19 October 2010

Accepted 24 October 2010

Available online 3 November 2010

\section{Keywords:}

Operational transfer path analysis

Force estimation

OPAX

Mount stiffness

\begin{abstract}
A B S T R A C T
Since its first publication in the beginning of the 1980s, transfer path analysis (TPA) has evolved into a widely used tool for noise and vibration troubleshooting and internal load estimation, for single source and multivariate problems. One of the main bottlenecks preventing its even more widespread use in the vehicle development process is the test time needed to build the full data model, requiring not only in-operation tests but also extensive frequency response function (FRF) measurements.

As a consequence, several new approaches, such as operational TPA, have appeared over the past years attempting to circumvent this limitation. These methods attract quite some attention as they only require operational data measured at the path references and target locations. However, despite being time-efficient, these methods suffer from several limitations that can lead to incorrect path contribution interpretations and wrong engineering decisions.

Hence, a new TPA approach is proposed, providing a good compromise between path accuracy and measurement time. The method is referred to as OPAX as it essentially uses inoperation data complemented with a minimal set of extra tests with forced excitation. The key idea of OPAX is the use of parametric models for identifying the operational loads. This makes the method scalable, enabling the engineer to use a simple model based on a small amount of measurement data for quick troubleshooting or increase accuracy using a more complex model together with additional measurements.
\end{abstract}

(c) 2010 Published by Elsevier Ltd.

\section{Introduction}

Transfer path analysis (TPA) is an experimental technique for identifying the vibro-acoustic transfer paths in a system. The energy transfer starts from the active system components that generate the structural and acoustic loads, and passes through the physical connections and along airborne pathways, to the response target locations in the passive system components. The acoustic and vibration responses at the target locations (e.g. interior noise, seat vibration, steering wheel vibration, etc.) are expressed as a sum of path contributions, each associated with an individual path and load. For example, for a target response $y_{k}(\omega)$ at point $k$, this is formulated in equation Eq. (1), where $y_{i k}(\omega)$ denotes the path contribution of path $i, \omega$ the frequency and $n$ the number of paths:

$$
y_{k}(\omega)=\sum_{i=1}^{n} y_{k i}(\omega)
$$

\footnotetext{
* Corresponding author. Tel.: +3216384417.

E-mail address: karl.janssens@lmsintl.com (K. Janssens).
} 
The first approaches to this problem used coherence analysis to assess $y_{i k}$, the contributions of the different inputs to the target, but such approaches suffered from problems related to separating partially correlated sources [1]. In the late 1980s, an alternative formulation based on a source-system-receiver model was developed for univariate noise and vibration problems. This formulation expresses each of the partial response contributions as the result of an individual structural or acoustic load acting at a localized interface, and a system response to this interface load [2-4]. The various contributions are added in a vector form to yield the global response. This procedure effectively corresponds to cutting the global system at the interface into an active part, generating the interface loads and a passive part reacting to them. For structural loads, this cut typically corresponds to the physical connection points (e.g. mounts, subsystem connections, etc.). For acoustic loads from vibrating surfaces or pulsations from nozzles or apertures, a discretization by omni-directional volume acceleration point sources is typically applied [5,6].

This systems' approach allows expressing each of the partial contributions explicitly as the result of the loads acting at each contribution location and the frequency response functions (FRF) between the load location and the considered target response. With the assumption that the system is linear and time invariant, this can be formulated as follows:

$$
y_{k}(\omega)=\sum_{i=1}^{n} H_{k i}(\omega) F_{i}(\omega)+\sum_{j=1}^{p} H_{k j}(\omega) Q_{j}(\omega)
$$

with $F_{i}(\omega)$ denoting $(i=1, \ldots, n)$ the structural interface loads or forces, $Q_{j}(\omega)(j=1, \ldots, p)$ the acoustic loads, typically volume velocities or accelerations, and $H_{i k}(\omega)$ and $H_{j k}(\omega)$ the uncoupled passive side FRFs from the input loads to the target.

Concise visualizations of the transfer path contribution results allow quick assessment of critical paths and frequency regions. The separation into loads and FRFs is furthermore the key to identify dominant causes and propose solutions (e.g. modify specific load inputs, a certain mount stiffness or a specific transfer path).

The test procedure to build a conventional TPA model typically requires two basic steps: (i) identification of the operational loads during in-operation tests (e.g. run-up and run-down) on the road or on a chassis dyno; and (ii) estimation of the uncoupled FRFs from excitation tests (e.g. hammer tests and shaker test), during which the active component is removed. The procedure is similar for both structural and acoustical loading cases, but the practical implementation is governed by the nature of the signals and loads.

With the advances in instrumentation the measurement of the FRFs between input loads and target response(s) is a wellcontrolled process. Direct measurement of the vibro-acoustic FRF may be subject to practical difficulties such as the fast and correct mounting of exciters so that in most cases the vibro-acoustic reciprocity principle [7,8] is utilized, allowing excitation at the target locations (e.g. cabin pressure in a vehicle) and the measurement of the structural response at the load contributing location (e.g. mount connection points). Reciprocal measurements are also the standard method for the measurement of the acoustic-acoustic FRFs in case of acoustic loads, since the number of target locations is typically much smaller than the number of load locations.

The main factor in the accuracy is the identification of the operational loads. Several approaches have been developed for both structural and acoustic loads. The present discussion is limited to the structural excitation case, but similar concepts are applicable to the acoustic load case [23]. Essentially, three ways exist to identify the forces [3,9-13]: direct measurement, mounts stiffness based identification and matrix inversion based identification.

The first approach is the direct measurement of the forces by dedicated devices such as load cells. Such direct measurement is however not possible in the majority of cases as the load cells require space and well-defined support surfaces, which often makes application impractical or even impossible without distorting the original connection.

The second approach is the mount stiffness method that can be used when the active and passive system components are connected through flexible mounts. This approach combines the differential operational responses across the mounts and the mount stiffness characteristics to estimate the transmitted forces. For a given mount, this can be expressed as follows:

$$
F_{i}(\omega)=K_{i}(\omega) \frac{\left(a_{a i}(\omega)-a_{p i}(\omega)\right)}{-\omega^{2}}
$$

with $F_{i}(\omega)$ denoting the mount force, $K_{i}(\omega)$ the dynamic mount stiffness and $a_{a i}(\omega)$ and $a_{p i}(\omega)$ the active and passive side mount accelerations, respectively. The mount stiffness method is a fast method, but its drawback is that accurate mount stiffness data is seldom available and even if it is, it is only valid for a given load condition and excitation amplitude.

The third approach is the inverse force identification method that identifies the operational loads $F_{i}(\omega)(i=1, \ldots, n)$ from nearby acceleration indicator responses $u_{q}(\omega)(q=1, \ldots, v)$ at the passive system side by multiplying these with the pseudoinverse of the measured force-acceleration FRF matrix between all force inputs and indicator responses. This can be described with the following equation:

$$
\left[\begin{array}{c}
F_{1}(\omega) \\
\vdots \\
F_{i}(\omega) \\
\vdots \\
F_{n}(\omega)
\end{array}\right]=\left[\begin{array}{ccccc}
H_{11}(\omega) & \cdots & H_{1 i}(\omega) & \cdots & H_{1 n}(\omega) \\
\vdots & \vdots & \vdots & \vdots & \vdots \\
H_{q 1}(\omega) & \cdots & H_{q i}(\omega) & \cdots & H_{q n}(\omega) \\
\vdots & \vdots & \vdots & \vdots & \vdots \\
H_{v 1}(\omega) & \cdots & H_{v i}(\omega) & \cdots & H_{v n}(\omega)
\end{array}\right]^{+}\left[\begin{array}{c}
u_{1}(\omega) \\
\vdots \\
u_{q}(\omega) \\
\vdots \\
u_{v}(\omega)
\end{array}\right]
$$

Matrix inversion and force identification are calculated frequency by frequency. The number of indicator responses $(v)$ must significantly exceed the number of forces $(n)$ - with a factor 2 as a rule of thumb - to minimize ill-conditioning problems 
when calculating the pseudo-inverse. The main disadvantage of this method is the large number of FRF measurements required to build the full matrix. This costs a lot of time and is the main bottleneck for more widespread industrial application.

The main industrial driver for innovations in TPA is hence the demand for simpler and faster methods. New methods such as Fast TPA and Multilevel TPA [14] partially address this demand reducing component loads to equivalent virtual locations, leading to globalized subsystem contributions. Other methods aim at reducing the amount of FRF measurements [15]. Another possibility for speeding up the TPA refinement process is characterizing the active component independently of its environment. This way no operational measurements are necessary after a modification of the passive subsystem [33]. The method, however, that recently attracted most attention is the operational path analysis approach [16,17], which has been referred to as OPA, OTPA, CTC, AMM or TPA FORM in different publications. Key to this method is that it requires only operational data measured at the path references (e.g. passive/active-side mount accelerations, pressures near vibrating surfaces, nozzles and apertures) and target points but no FRFs. Essentially, it is a transmissibility method as known from structural dynamics [18], characterizing the co-existence relationship between the target response(s) and path references. This method is indeed very time-efficient and offers very interesting perspectives to get a quick insight in the noise and vibration patterns of the problem under investigation.

However, its use as a true modeling and design engineering tool, as is TPA, is subject to several limitations that require caution when interpreting the obtained results [19,23]. One critical factor is the potential cross-coupling between the assumed path references. Because of the system's modal behavior, a single force in one of the mounts causes vibrations at all locations of the passive-side structure, hence also at the locations of other path inputs. Consequently, a high response level at a path input location does not necessarily imply that a force or acoustic load is entering the system at that location. Co-existence of signals does not imply causality. This cross-coupling effect hence may lead to an incorrect interpretation in terms of significant paths. Next to this, the method may suffer from ill-conditioning problems, such as rank deficiency at resonances, related to estimating the transmissibilities from operational data as it has been reported in [32,33]. This is dependent on the dimensionality of the primary noise problem and has an impact on the required extent of the test conditions [20]. Although the use of SVD techniques may improve the quality of estimation, in particular in case of noisy data, the inherent breakdown of dimensionality may still result in unreliable transmissibility estimates in many situations. This is one of the aspects to be monitored when validating the analysis results. Finally, there are potential errors due to missing paths in the analysis. Simulations have shown [21] that the contributions of missing paths are distributed over the other ones, introducing errors that are often hard to recognize. Due to the backward-forward use of the same data, a good synthesis of summed contributions is not representative for completeness and quality of the results.

This publication introduces a new TPA method, which combines the efficiency of OPA and the effectiveness of the existing conventional TPA methods. The key idea of the new method is the use of parametric models for identifying the operational loads. This makes the method scalable, enabling the engineer to use a simple model based on a small amount of measurement data for a quick troubleshooting or increase accuracy using a more complex model together with additional measurements. This new TPA method is referred to as operational path analysis with eXogeneous inputs (OPAX) in the following discussion, where the "X"-suffix refers to the use of additional excitation-based measurement data to estimate the parametric models. The name OPAX also makes the association with the ARX type of parametric models (e.g. ARX, ARMAX, etc.) [24], which are well known and widely used in system identification.

\section{OPAX method}

The OPAX method differs from the existing ones in the identification of the operational loads. The key is the use of parametric models characterizing the operational forces and acoustic loads as a function of measured path inputs such as mount accelerations and acoustic pressures. The parametric load models are estimated from (i) in-situ measured operational path inputs and target response signals and (ii) transfer path FRFs using mathematical techniques, such as, for example, the least squares (LS) estimation approach. Extra acceleration and/or pressure indicators can be included in the set of equations to obtain more robust parameter estimations, but this is optional.

A schematic representation of the different variables to be measured and identified is given in Fig. 1. The figure presents a system with an active part generating forces $F_{i}(\omega)(i=1, \ldots, n)$ and acoustic loads $Q_{j}(\omega)(j=1, \ldots, p)$ and with a passive part reacting to these loads. A typical example of such a system is a vehicle body on which a powertrain is mounted. In this example, the powertrain forms the active part of the system, while the body with passenger compartment is the passive part.

In order to identify the noise transfer paths from the active system part, through the physical connection elements and along airborne pathways, to the target(s) of interest, the following variables are typically measured:

- Operational measurements:

O Responses:

- Target(s): pressures and/or accelerations $y_{k}(\omega)(k=1, \ldots, u)$.

- Optionally some extra indicators, for example, some acceleration indicators closeby the mounts: $u_{q}(\omega)(q=1, \ldots, v)$.

○ Path inputs:

- Structural path inputs: active and passive-side accelerations at the mount connections $a_{a i}(\omega)(i=1, \ldots, n)$ and $a_{p i}(\omega)(i=1, \ldots, n)$.

- Acoustic path inputs: pressures near the acoustic sources $p_{j}(\omega)(j=1, \ldots, p)$. 


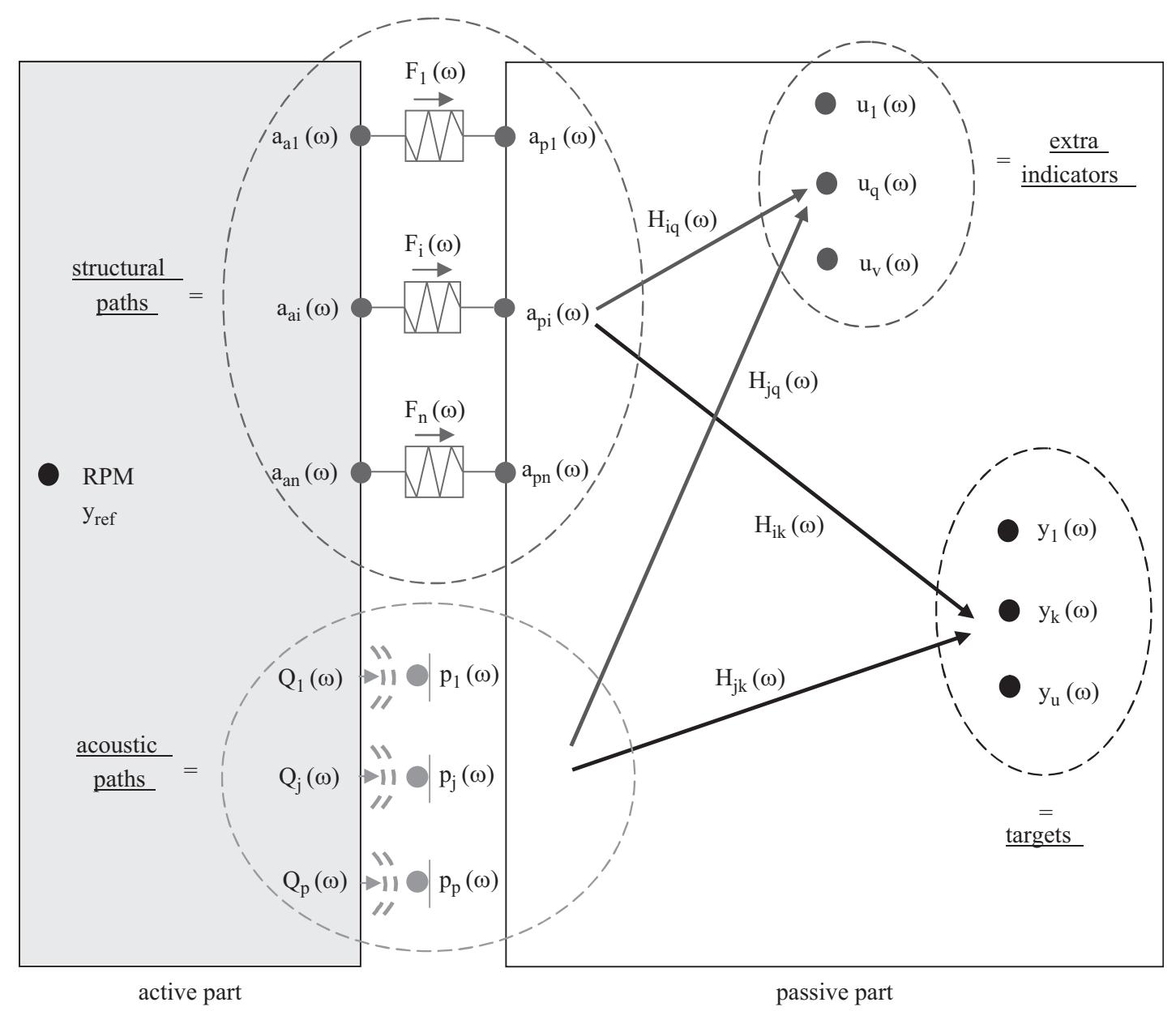

Fig. 1. Schematic representation of a system with an active component generating forces and acoustic loads, and a passive component responding to these loads. Operational data (path inputs, targets and extra indicators) and FRFs are measured for an OPAX analysis.

Tacho signal (pulse train, RPM) or phase reference

- FRF measurements:

FRFs from loads to target(s): $H_{k i}(\omega), H_{k j}(\omega)$.

FRFs from loads to extra indicators when used: $H_{q i}(\omega), H_{q j}(\omega)$

OPAX comprises 5 major steps. A flow diagram of the method is shown in Fig. 2. The steps are as follows:

- Phase 1: operational measurements

First, operational measurements are performed to acquire the time data of all measurement channels. These can be several run-up or run-down measurements at different conditions (e.g. for various throttles, gears, etc.) but even a single measurement can already be sufficient. It depends on the condition(s) for which the OPAX model is to be developed. During the operational measurements, all mount accelerations and pressure inputs and all responses at the target(s) and indicators are measured synchronously. Order envelopes (phase and amplitude in function of rotational speed) are then tracked for all measured input and response channels. Strictly speaking, only the orders of interest must be processed. However, the more orders are used for identifying the parametric load models, the more robust the estimation becomes and the more accurate path contributions can be derived. After the measurements one ends up with complex order data of all measured channels.

- Phase 2: FRF measurements

In the second phase, the uncoupled FRFs are measured between the input loads and the target response(s). The FRFs can be measured in a direct or reciprocal way. The use of reciprocal measurements (excitation at the target location(s) and responses measured at the interface) has two advantages: (i) only one excitation is needed per target point while the direct approach requires one excitation per input load; (ii) the limited space at the path inputs can lead to direction errors in the direct FRF measurements of up to $10 \mathrm{~dB}$ [22]. In case additional indicators are used, the FRFs from the inputs to the indicators must also be measured. The sequence of phases 1 and 2 may be interchanged. 


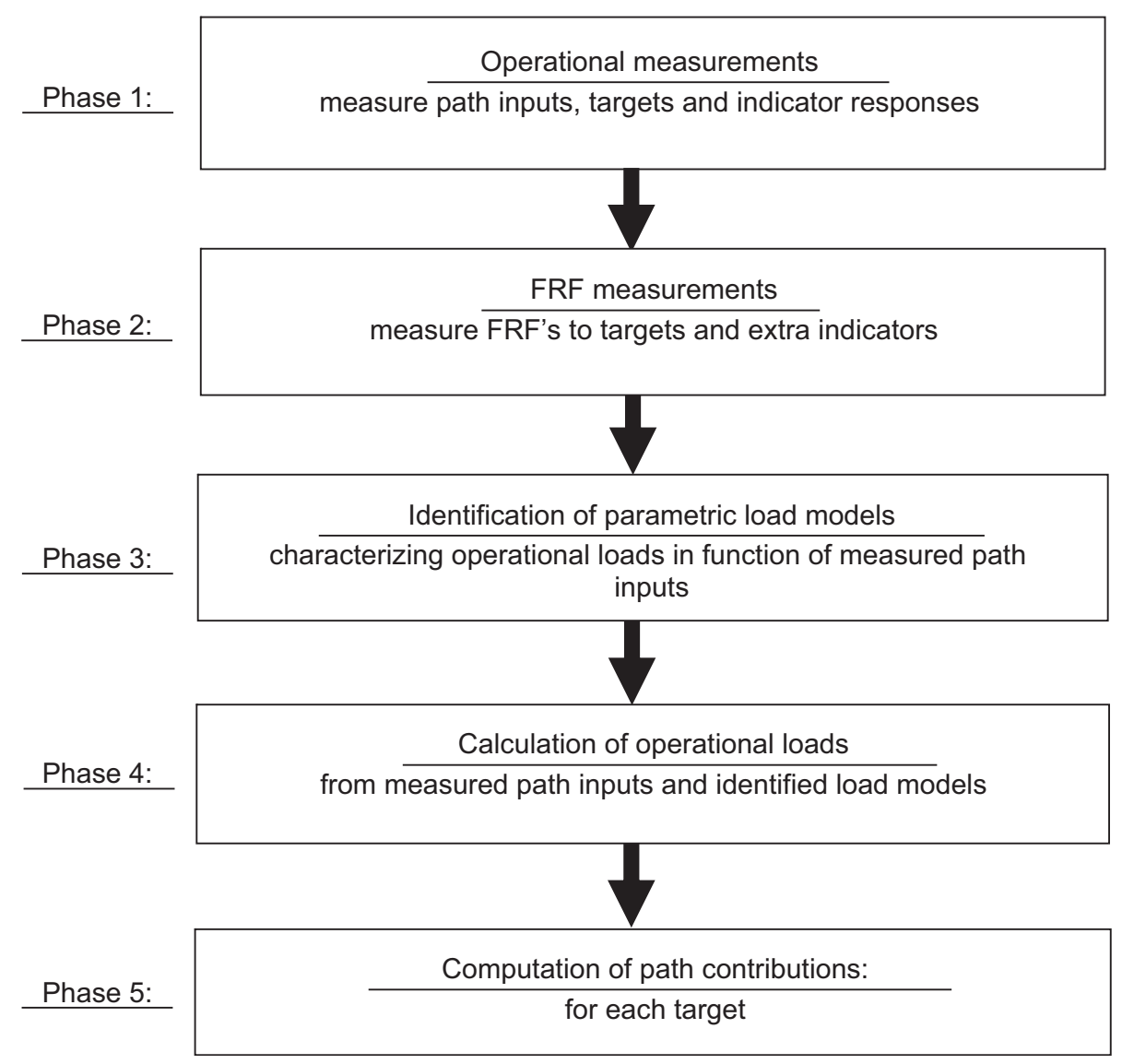

Fig. 2. Flow diagram of the OPAX approach, comprising 5 major steps.

- Phase 3: identification of parametric load models

Phase 3 forms the key step of the method. In this phase, the operational forces and acoustic loads are identified in a parametric way from the measurement data obtained in phases 1 and 2 . The advantage of this approach is that these parameters are frequency independent which, as shown in the following steps, reduces the complexity of the problem. First, the parametric load models are formulated, describing the forces and acoustic loads as a function of the acceleration and pressure path inputs:

$$
\begin{aligned}
& F_{i}(\omega)=f\left(\text { parameters, } a_{a i}(\omega), a_{p i}(\omega)\right) \\
& Q_{j}(\omega)=g\left(\text { parameters, } p_{j}(\omega)\right)
\end{aligned}
$$

Substituting these parametric load models in the source-transfer-response equations for the target(s) and extra indicators, we obtain a new set of equations with these parameters as unknowns:

$$
\begin{aligned}
& y_{k}(\omega)=\sum_{i=1}^{n} H_{k i}(\omega) F_{i}\left(\text { parameters, } a_{a i}(\omega), a_{p i}(\omega)\right)+\sum_{j=1}^{p} H_{k j}(\omega) Q_{j}\left(\text { parameters }, p_{j}(\omega)\right) \\
& u_{q}(\omega)=\sum_{i=1}^{n} H_{q i}(\omega) F_{i}\left(\text { parameters, } a_{a i}(\omega), a_{p i}(\omega)\right)+\sum_{j=1}^{p} H_{q j}(\omega) Q_{j}\left(\text { parameters }, p_{j}(\omega)\right)
\end{aligned}
$$

with $H_{k i}(\omega)$ and $H_{q i}(\omega)$ the FRFs from loads to targets and indicators, respectively, measured reciprocally or though direct excitation (Step 2), and $y_{k}(\omega)$ and $u_{q}(\omega)$ the complex order data of targets and indicators, respectively, as measured in Step 1.

This OPAX formulation yields a large overdetermined set of equations. The unknown variables of this system of equations are the model parameters, while the known variables are the measured FRFs and frequency values. The structure of the equations and the nature of the unknown parameters directly depend on the type of load models used in the formulation (e.g. Eq. (8) or (9)), which can be either linear or non-linear. Solving the system of equations the model parameters are identified, and Eq. (7) expresses the relationship between the measured path reference signals, $a_{a i}, a_{p i}$ and $p_{j}$ and the target responses, $y_{k}$ and $u_{q}$. 


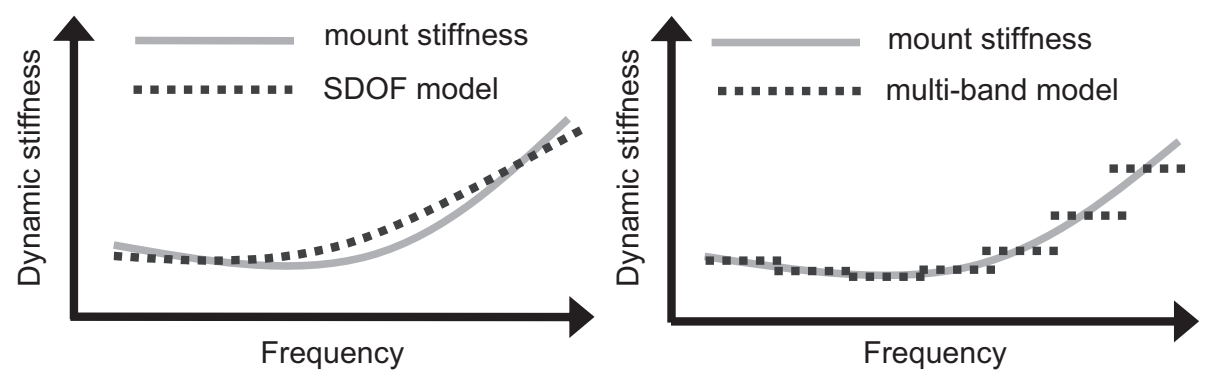

Fig. 3. Examples of OPAX mount models: (left) SDOF model and (right) multi-band model.

Any suitable mount or source model can be used as the parametric model in Eqs. (5) and (6). In this paper only two basic models will be introduced. The first one, for structural forces acting in mounts, is a single degree of freedom (SDOF) model:

$$
F_{i}(\omega)=K_{i}(\omega) \frac{\left(a_{a i}(\omega)-a_{p i}(\omega)\right)}{-\omega^{2}}
$$

where

$$
K_{i}(\omega)=-m_{i} \omega^{2}+j c_{i} \omega+k_{i}
$$

$m_{i}, c_{i}$ and $k_{i}$ are physically meaningful parameters representing the dynamic mass, damping and static stiffness of a given mount, respectively. A schematic representation of the SDOF model is shown in Fig. 3(a).

An interesting alternative to the previous model is, for example, the application of a multi-band model, assuming a constant complex mount stiffness for a given frequency band. This model is useful in the case when the mount or acoustic source behavior is not known a priori. The idea of such a bandwise approximation of the dynamic mount stiffness is shown in Fig. 3(b).

$$
K_{i}(\omega)=k_{i}
$$

For the acoustic loads, a multi-band model description may also be used, assuming a bandwise constant relation between the load and the measured path input. The mathematical formulation is as follows:

where $Q_{j}(\omega)=\theta_{j}(\omega) p_{j}(\omega)$

$$
\theta_{j}(\omega)=\theta_{j}
$$

is a constant complex value within each frequency band.

Since the structure of the resulting linear system of equations strongly depends on the choice of model, a more detailed description is provided separately, in Section 3, examining two typical user scenarios.

As further discussed in Section 4, a priori known parameters (e.g. static stiffness properties $k_{i}$ of some mounts may be known) or known relations among parameters (e.g. some mounts may have equal stiffness properties in $x$-and $y$-directions) can be taken into account, reducing the number of model parameters and improving the conditioning of the set of equations to be solved. Furthermore, in general, adding more input data, i.e. more orders, targets and indicators, the accuracy of the model parameter estimation can be further improved, with the condition that the added data is different from, and is not noisier than, the data already contained in the model. Finally, the use of balancing factors to scale (i) the order components, (ii) the structural and acoustic terms and (iii) the target and indicator responses will also help to improve the parameter estimations.

- Phase 4: calculation of operational loads

In phase 4 , the operational input forces $(i=1, \ldots, n)$ and volume accelerations $(j=1, \ldots, p)$ are determined by substituting the estimated model parameter values in Eqs. (5) and (6). The loads are typically calculated per order component.

- Phase 5: computation of path contributions

Finally, once the operational loads are identified, the path contributions can be calculated for each target point $k$, multiplying the loads with the corresponding FRF, as expressed in Eq. (2). Visualizations of the path contribution results then allow the assessment of critical paths, order components and frequency regions, providing a basis for modifications of, for instance, mount characteristics or path FRFs.

\section{OPAX user scenarios}

The structural and acoustic path contributions in OPAX can be identified either separately or simultaneously. These two typical user scenarios and their mathematical formulation are presented in the sections below.

\subsection{Separate identification of structural path contributions}

Similar to the TPA matrix inversion method, the structural path contributions to the target(s) can be identified without considering the acoustic loads. For this, a number of acceleration indicators $u_{q}(\omega)$ need to be measured near the mounts. It can 
be assumed that these indicators are hardly affected by the acoustic loads, hence the following formulation can be written for $u_{q}(\omega)$ :

$$
u_{q}(\omega)=\sum_{i=1}^{n} H_{q i}(\omega) F_{i}(\omega)=\sum_{i=1}^{n} H_{q i}(\omega) K_{i}(\omega) \frac{\left(a_{a i}(\omega)-a_{p i}(\omega)\right)}{-\omega^{2}}
$$

\subsubsection{SDOF mount model}

Assuming the SDOF mount model of Eq. (9) with real mass $m_{i}(i=1, \ldots, n)$, damping $c_{i}(i=1, \ldots, n)$ and stiffness $k_{i}(i=1, \ldots, n)$, we obtain

$$
u_{q}(\omega)=\sum_{i=1}^{n} H_{q i}(\omega)\left(-m_{i} \omega^{2}+j c_{i} \omega+k_{i}\right) \frac{\left(a_{a i}(\omega)-a_{p i}(\omega)\right)}{-\omega^{2}}=\sum_{i=1}^{n}-m_{i}\left(\omega^{2} G_{q i}(\omega)\right)+c_{i}\left(j \omega G_{q i}(\omega)\right)+k_{i} G_{q i}(\omega)
$$

where

$$
G_{q i}(\omega)=H_{q i}(\omega) \frac{\left(a_{a i}(\omega)-a_{p i}(\omega)\right)}{-\omega^{2}}
$$

In matrix notation, this gives

$$
\left[\begin{array}{lllll}
\cdots & -\omega^{2} G_{q i}(\omega) & j \omega G_{q i}(\omega) & G_{q i}(\omega) & \cdots
\end{array}\right]\left[\begin{array}{c}
\vdots \\
m_{i} \\
c_{i} \\
k_{i} \\
\vdots
\end{array}\right]=\left[\begin{array}{ll}
u_{q}(\omega)
\end{array}\right]
$$

Considering a typical automotive application case with data consisting of $m$ orders and $r$ RPM points per order, the following system of equations can be formulated:

$$
\left[A_{q}\right][X]=\left[B_{q}\right]
$$

where

$$
\begin{aligned}
& \left.\left[A_{q}\right]=\mid \begin{array}{||ccccc}
\cdots & -\omega_{11}^{2} G_{q i}\left(\omega_{11}\right) & j \omega_{11} G_{q i}\left(\omega_{11}\right) & G_{q i}\left(\omega_{11}\right) & \cdots \\
\cdots & -\omega_{21}^{2} G_{q i}\left(\omega_{21}\right) & j \omega_{21} G_{q i}\left(\omega_{21}\right) & G_{q i}\left(\omega_{21}\right) & \cdots \\
\vdots & \vdots & \vdots & \vdots & \vdots \\
\cdots & -\omega_{r 1}^{2} G_{q i}\left(\omega_{r 1}\right) & j \omega_{r 1} G_{q i}\left(\omega_{r 1}\right) & G_{q i}\left(\omega_{r 1}\right) & \cdots \\
\cdots & -\omega_{12}^{2} G_{q i}\left(\omega_{12}\right) & j \omega_{12} G_{q i}\left(\omega_{12}\right) & G_{q i}\left(\omega_{12}\right) & \cdots \\
\cdots & -\omega_{22}^{2} G_{q i}\left(\omega_{22}\right) & j \omega_{22} G_{q i}\left(\omega_{22}\right) & G_{q i}\left(\omega_{22}\right) & \cdots \\
\vdots & \vdots & \vdots & \vdots & \vdots \\
\cdots & -\omega_{r 2}^{2} G_{q i}\left(\omega_{r 2}\right) & j \omega_{r 2} G_{q i}\left(\omega_{r 2}\right) & G_{q i}\left(\omega_{r 2}\right) & \cdots \\
\vdots & \vdots & \vdots & \vdots & \vdots \\
\cdots & -\omega_{1 m}^{2} G_{q i}\left(\omega_{1 m}\right) & j \omega_{1 m} G_{q i}\left(\omega_{1 m}\right) & G_{q i}\left(\omega_{1 m}\right) & \cdots \\
\vdots & \vdots & \vdots & \vdots & \vdots \\
\cdots & -\omega_{2 m}^{2} G_{q 1}\left(\omega_{2 m}\right) & j \omega_{2 m} G_{q i}\left(\omega_{2 m}\right) & G_{q i}\left(\omega_{2 m}\right) & \cdots \\
\cdots & -\omega_{r m}^{2} G_{q 1}\left(\omega_{r m}\right) & j \omega_{r m} G_{q i}\left(\omega_{r m}\right) & G_{q i}\left(\omega_{r m}\right) & \cdots
\end{array}\right]=\text { order } 1
\end{aligned}
$$

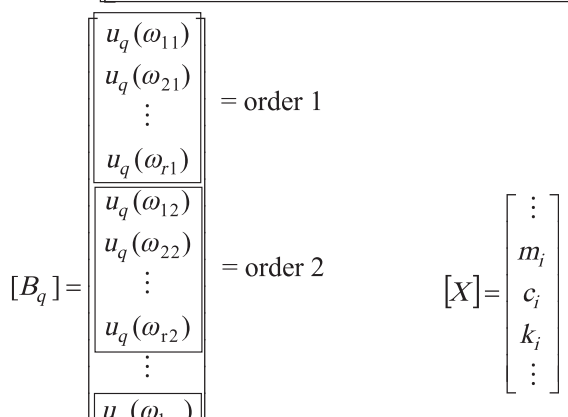

$$
\begin{aligned}
& u_{q}\left(\omega_{2 m}\right) \\
& u_{q}\left(\omega_{r m}\right)
\end{aligned}
$$

where $\omega_{x y}=o_{y} \operatorname{RPM}_{x} / 60$, with $o_{y}$ being the order number $(y=1, \ldots, m)$ and $\operatorname{RPM}_{x}$ the rotational speed $(x=1, \ldots, q)$.

For all indicator points $u_{q}(q=1, \ldots, v)$, Eq. (17) becomes

$$
[A][X]=[B]
$$


where

$$
[A]=\left[\begin{array}{c}
A_{1} \\
\vdots \\
A_{v}
\end{array}\right] \text { and }[B]=\left[\begin{array}{c}
B_{1} \\
\vdots \\
B_{v}
\end{array}\right]
$$

The least squares (LS) solution of this linear system of equations is

$$
[X]=\left[\begin{array}{l}
\operatorname{Re}(A) \\
\operatorname{Im}(A)
\end{array}\right]^{+}\left[\begin{array}{l}
\operatorname{Re}(B) \\
\operatorname{Im}(B)
\end{array}\right]
$$

where $[X]$ is the solution vector with real estimated mass $m_{i}$, damping $c_{i}$ and stiffness $k_{i}$ values, and " " " denotes the pseudo inverse, which is calculated with the help of singular value decomposition. Due to the significant overdetermination of the system of equations, the condition number is generally low and no truncation is applied during the inversion.

Substituting these parameter values in Eqs. (8) and (9), we obtain the dynamic mount stiffness profiles and operating forces. Multiplying these with the according transfer path FRFs gives the structural path contributions to the target(s) of interest:

$$
y_{k}(\omega)=\sum_{i=1}^{n} y_{k i}(\omega)=\sum_{i=1}^{n} H_{k i}(\omega) F_{i}(\omega)
$$

\subsubsection{Multi-band model}

Instead of the SDOF model, one can also apply the multi-band formulation of Eq. (10), which does not assume SDOF behavior of the mounts. In this case, the frequency range is split into a number of adjacent or partly overlapping bands and a system of equations is formulated and solved for each band. The indicator responses within a certain frequency band, with $\omega_{z, \max }$ and $\omega_{z, \min }$ as upper and lower frequency limits, respectively, can now be expressed as follows:

$$
u_{q}(\omega)=\sum_{i=1}^{n} H_{q i}(\omega) F_{i}(\omega)=\sum_{i=1}^{n} H_{q i}(\omega) k_{i} \frac{\left(a_{a i}(\omega)-a_{p i}(\omega)\right)}{-\omega^{2}}=\sum_{i=1}^{n} k_{i} G_{q i}(\omega)
$$

where

$$
G_{q i}(\omega)=H_{q i}(\omega) \frac{\left(a_{a i}(\omega)-a_{p i}(\omega)\right)}{-\omega^{2}}
$$

Formulating this equation for all orders and RPM points in a given frequency band results in the same linear system of equation as Eq. (17), where the $A_{q}$ and $B_{q}$ matrices only contain information between the upper and the lower limits of the band: $\omega_{z, \min } \leq \omega_{x y} \leq \omega_{z, \max }$.

In case of a mount exhibiting non-linear behavior, e.g. in function of the load, the mount could be linearized for a number of load regions. Then, this model type could be used to separately estimate the parameters for these different load regions, which could be separated based on the RPM since the torque of an internal combustion engine is linked to its RPM.

For all indicators together an overdetermined set of equations is obtained again, just as Eq. (18), which can be solved in a LS manner. In this case the solution vector contains the complex mount stiffness estimates for the considered frequency band.

The above equations Eqs. (21) and (22) can be formulated and solved for all predefined frequency bands. By doing so, we obtain the dynamic mount stiffness profiles for the complete frequency range of the OPAX analysis under study. Finally, by combining them with the operational mount accelerations and the according transfer path FRFs, we obtain the path contributions for the targets of interest.

The principle of the multi-band mount stiffness estimation is schematically represented in Fig. 4. One can easily understand that the wider the frequency bands are and the more the orders and indicator points are included, the more overdetermined the system of equations becomes resulting in more robust estimation of the mount stiffness values. This is an important consideration that will be further discussed in Section 4.

It is important to mention that for rigid connections, typically only the passive side accelerations are considered, since the differential response across these connections is very small. The multi-band formulation in Eqs. (21) and (22) still holds here, but the parameters $k_{i}(i=1, \ldots, n)$ in the solution vector are to be interpreted as local body stiffnesses at the mount connection points. In such cases, relatively small frequency bands need to be used to estimate the local body stiffnesses, but by choosing narrower frequency bands, more parameters need to be estimated, therefore more data is required to avoid ill-conditioning problems. In the extreme case, when using (i) frequency bands of a only one spectral line and (ii) only one single order, the OPAX formulation is equivalent to TPA matrix inversion,

\subsection{Simultaneous identification of structural and acoustic path contributions}

The structural and acoustic path contributions can also be estimated simultaneously from the target(s) only, without using extra indicator points. In this case only one reciprocal FRF measurement is required per target location, reducing the measurement time. This fast OPAX scenario is very useful for a quick identification of the critical paths. 

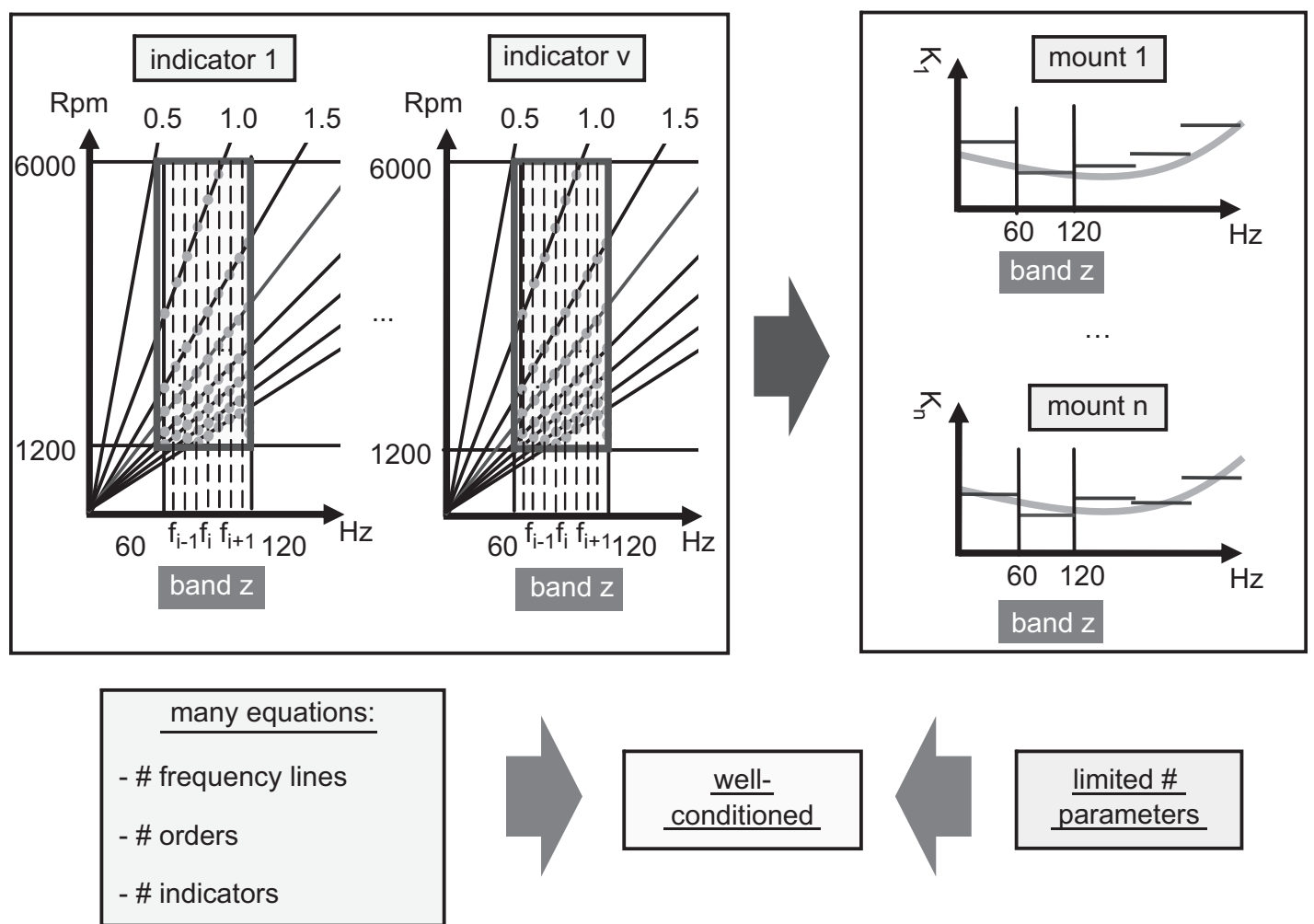

Fig. 4. Principle of multi-band mount stiffness estimation.

If we take, for example, the multi-band formulation of Eqs. (10) and (12) and divide the frequency range in a number of bands, then the target responses in each of the bands can be expressed as follows:

$$
\begin{aligned}
y_{k}(\omega) & =\sum_{i=1}^{n} H_{k i}(\omega) F_{i}(\omega)+\sum_{j=1}^{p} H_{k j}(\omega) Q_{j}(\omega)=\sum_{i=1}^{n} H_{k i}(\omega) k_{i} \frac{\left(a_{a i}(\omega)-a_{p i}(\omega)\right)}{-\omega^{2}}+\sum_{j=1}^{p} H_{k j}(\omega) \theta_{j} p_{j}(\omega) \\
& =\sum_{i=1}^{n} k_{i} G_{k i}(\omega)+\sum_{j=1}^{p} \theta_{j} G_{k j}(\omega)
\end{aligned}
$$

where

$$
\begin{aligned}
& G_{k i}(\omega)=H_{k i}(\omega) \frac{\left(a_{a i}(\omega)-a_{p i}(\omega)\right)}{-\omega^{2}} \\
& G_{j k}(\omega)=H_{k j}(\omega) p_{j}(\omega)
\end{aligned}
$$

In matrix form and for all orders and RPMs, this again gives an overdetermined set of equations:

$$
\left[A_{k}\right][X]=\left[B_{k}\right]
$$

where

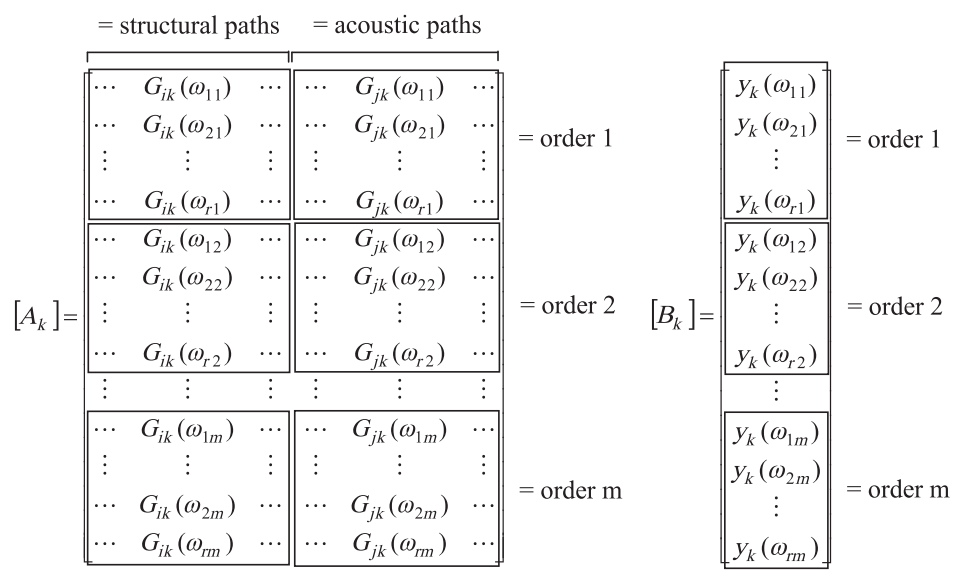




$$
\left.[X]=\left[\begin{array}{c}
\vdots \\
k_{i} \\
\vdots \\
\vdots \\
\theta_{j} \\
\vdots
\end{array}\right]\right]=\text { structural paths }
$$

and

$$
\omega_{z, \min } \leq \omega_{x y} \leq \omega_{z, \max }
$$

The first $n$ columns in matrix $\left[A_{k}\right]$ represent the structural paths, while the last $p$ columns are the acoustic paths. Writing down this system of equations for all targets $y_{k},(k=1, \ldots, u)$, we again obtain a system of equations as in Eq. (18). Solution of this system of equations yields the mount stiffness estimates $k_{i}(i=1, \ldots, n)$ and acoustic point mobilities $\theta_{j}(j=1, \ldots, p)$.

However, in this OPAX scenario, the structural and acoustic columns of matrix $[A]$ are typically a few orders of magnitude different, which can lead to incorrect estimation of the parameters. In order to avoid this, a structural-acoustic balancing is applied, rescaling the structural and acoustic blocks of columns in matrix $[A]$ to the same RMS level.

\subsection{Overdetermination using multiple indicators/targets}

In both user scenarios presented in Sections 3.1 and 3.2, multiple indicators and/or targets can be used to improve the estimation of the model. Even though there are no theoretical obstacles to such an approach, in practice it could be expected that different combinations of indicators/targets yield differing model estimations. For this reason it is worth to discuss this aspect of the OPAX method in more depth.

In an ideal case, with a noiseless and error free linear time invariant system, even one well-chosen target could be sufficient for the proper identification of the models. The deviation of the estimated models in an actual measurement has two sources: measurement errors and the observability of the structure. The former is actually the reason why different indicators/targets are combined in OPAX, e.g. by stacking the indicator data in [B] as, e.g. in Eq. (17), since this allows finding the best model estimate in a statistical sense. With respect to the latter, even in an ideal, noiseless measurement, badly chosen sensor locations would have low observability, that is, only a few modes would affect the sensor. Combining data from such sensors helps to ensure that all relevant modes are observable by the sensor, improving the conditioning of the system matrix. This actually leads us to the problem selection of sensor locations, which is addressed below, in Section 4.2.

\section{OPAX considerations}

OPAX essentially comes down to solving a model identification problem. Three important aspects have to be taken into account: (1) the structure of the model, (2) the number of model parameters to be estimated and (3) the amount of information available to identify the model. The first one must be chosen in a way such that it adequately describes the operational loads. Secondly, the number of these parameters depends on the number of transfer paths and the complexity of the model itself. And thirdly, the amount of information in the measurement data depends on the number of targets, the number of additional indicators, the number of order components, the RPM range and the system's modal behavior. All of these co-dependently influence the quality of the OPAX analysis. In the light of this, the effect of these aspects, namely (i) the number of response points, (ii) the use of single or multiple orders, (iii) the type of model and (iv) the use of a priori information are considered in the following sections.

\subsection{Number of response points}

The choice of the amount of response points, the number of targets and extra indicators - considering a fixed set of the other three aspects - is a trade-off between measurement speed and accuracy. In many cases, simple parametric load models and resulting path contributions can be identified sufficiently well even from one target only without the need for extra indicators or targets. This would require only one reciprocal FRF measurement, thus making OPAX fast and suitable for a quick $\mathrm{NVH}$ troubleshooting. Of course such a measurement identifies the most critical problems but does not provide detailed insight into the behavior of the system. To achieve the latter, more targets and indicators need to be added increasing the information content of the measurements. In this case more refined models can be used and more robust and detailed results will be achieved. It is worth mentioning that even then the FRF measurement efforts remain limited compared to the TPA matrix inversion method.

\subsection{Selection of response point locations}

The question of finding the best locations for the overdetermination point locations is not unique to the OPAX method, but a general problem in structural dynamics. Articles on this topic have been published, e.g. in the context of model updating $[26,27]$, structural control $[28,29]$, but also with respect to inverse force identification $[13,30,31]$. The proper selection of 
sensor locations is fundamentally linked to the observability of the system: on one hand, the selected indicator locations should cover the global contributing modes, and on the other hand, they should be well distributed over the structure to capture local phenomena. The controllability of the system also plays a role in the analysis, since the improvement that can be achieved by modifying a certain path depends on the amount of control the given path input exerts over the target. The drawback of existing methods is that they require a priori knowledge about the structure, which is often not available in TPA measurements. In general, the following simple guidelines should be followed for the placement of OPAX indicators: (i) the indicator sensors should not be located in the close vicinity of the path input sensors or of each other, since in these cases they would provide redundant information and (ii) experience indicates that at least one indicator for each of the measurement coordinate axes (e.g. $x, y, z)$ should be included in the analysis.

\subsection{Single or multiple orders}

As discussed before, multiple orders can be included in the OPAX system of equations. By doing so, the conditioning of the system can be improved, reducing the error on the parameter estimates. This is recommended in case of a quick OPAX measurement having only a limited number of response points. In such instances order balancing is typically applied, to avoid the LS estimation becoming dominated by the strongest orders. Mathematically, this is achieved by multiplying the order blocks in the matrix $[A]$ and response vector $[B]$ with a scaling factor in such a way that all response orders are set to the same RMS value. This balancing makes sure that all orders add information to the system of equations, improving the condition number. However, one must be careful with including weak orders and rescaling them to the same level as the stronger ones. These weak orders typically contain large errors as they are hard to track, hence rescaling them will amplify these errors and deteriorate the LS estimation.

Of course, just as in traditional TPA matrix inversion, the OPAX model parameters and operating loads can also be estimated order by order. In this way, load dependent mount stiffness profiles are estimated, which is closer to reality. The drawback is that the number of equations is much lower than in the multi-order case, which has to be compensated by measuring additional indicators in order to have a proper conditioning at the cost of increased FRF measurement efforts.

\subsection{Type of model: SDOF or multi-band}

The type of model is also an important factor. Two types of OPAX models were introduced earlier in this paper: (i) the SDOF model and (ii) the multi-band model. Both models have their advantages and disadvantages:

- SDOF model:

The SDOF model is a suitable model for soft mounts, as many of them show an SDOF-like behavior.

$\circ$ It is a physical model with physically meaningful mass $\left(m_{i}\right)$, damping $\left(c_{i}\right)$ and stiffness $\left(k_{i}\right)$ parameters.

$\bigcirc$ This type of model is not appropriate for hydraulic mounts that have a more complex behavior, for rigid connections or acoustic loads.

- Multi-band model:

The multi-band model is applicable to any type of soft mount, rigid connection and acoustic load. It does not assume SDOF behavior.

It is more of a black box model; the parameters have limited physical meaning.

- The dynamic stiffness of the soft mounts and the driving point relations for the rigid connections and acoustic loads are assumed to be bandwise constant in pre-defined frequency bands. Hence, approximation errors are made which become larger when taking wider frequency bands.

For soft mounts in general, relatively wide frequency bands can be chosen up to a $100 \mathrm{~Hz}$ bandwidth or even more without making a significant approximation errors. Of course, this error can always be reduced by taking smaller bands, but more indicators will then be required to have a well-conditioned system of equations. In case of a quick OPAX measurement without extra indicators on a system with a large number of paths, it is likely that wider bands than optimal have to be used to avoid ill-conditioning, and this brings along reduced path accuracy.

- For rigid connections and acoustic loads, smaller frequency bands are recommended in order to properly characterize the driving point behavior. The smaller the frequency bands, the better the model description, but more indicators are then required for a proper estimation of the parameters. The extreme case is the use of a single frequency line per bandwidth, transforming the OPAX formulation into a classical TPA matrix inversion, thus requiring twice as many indicators as paths.

In Section 6 further guidelines are provided for choosing the parameters, supported by simulation examples.

\subsection{Use of a priori information}

A priori information on the mounts can be included in the OPAX set of equations. 
First of all, known parameter values can simply be substituted. For example, using the SDOF model formulation of Eq. (17) and knowing the dynamic mass $\left(m_{i}\right)$ and static stiffness $\left(k_{i}\right)$ of the mounts, the $m_{i}$ - and $k_{i}$-related terms can be substituted in the right side of Eq. (17), giving a reduced system of equations with only the damping coefficients $\left(c_{i}\right)$ as unknown parameters.

Another possibility is that certain relations between the mounts or between the three directions within a mount are known. For example, applying a multi-band model as in Eqs. (21) and (22) and knowing that the mount stiffnesses in the radial directions $x$ and $y$ are equal and twice as soft as in the axial direction $z$, then the $x$-, $y$-and $z$-terms of each mount can be linearly combined again yielding a reduced number of unknowns. Solving this reduced system, an estimate for the stiffnesses in the axial direction is obtained from which the stiffnesses in the radial directions can then be derived. Also, linear relations between different mounts can be accounted for, e.g. when two mounts are known to have the same stiffness characteristics.

If a priori information is available and reliable, its use is recommended. It reduces the system of equations, allowing a better estimation of the remaining unknown parameters.

\section{OPAX scalability and quality indicators}

\subsection{The scalability of OPAX}

Based on the aspects discussed above it is easy to see an advantage of this new TPA approach, namely that the use of parametric load models makes the OPAX method scalable, enabling the engineer to use a simple model based on a small amount of measurement data for a quick troubleshooting and to increase accuracy using a more complex model together with additional measurements. This scalability is schematically represented in Fig. 5, indicating the number of parameters to be estimated for different types of models.

On one side we have the mount stiffness TPA method where all mount stiffness characteristics are known and no parameters need to be estimated. This can be considered as a limiting case of OPAX where a complete set of a priori information is available.

On the other side, using a multi-band model with frequency bands of one spectral line and only one order for identifying the model parameters, the OPAX approach will converge to the matrix inversion TPA method. A large set of parameters need to be estimated in this case requiring a large number of indicators that should exceed the number of paths with a factor of 2 as a rule of thumb.

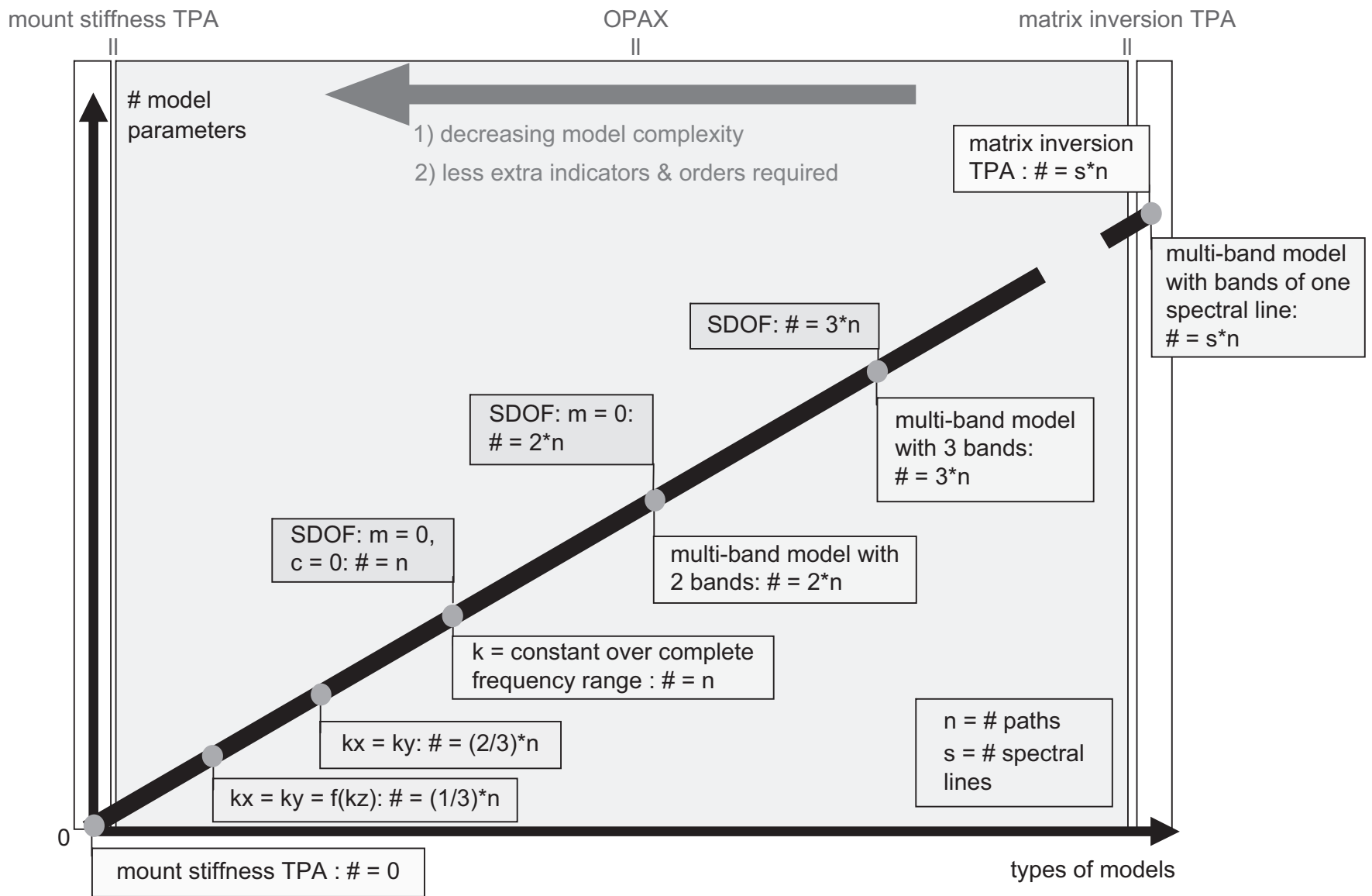

Fig. 5. OPAX scalability. 
OPAX covers the complete range between these two extremes, with the low-parameterized models on the left side (e.g. multi-band models with wide frequency bands, SDOF models with a priori known parameter values, models with known parameter relations, etc.) and the more complex models on the right side (e.g. multi-band models with small frequency bands, etc.).

This scalability is the main strength of OPAX, giving it great flexibility. It is no longer necessary to know all mount stiffness characteristics or measure the full matrix of FRFs to run a mount stiffness or a matrix inversion TPA analysis. OPAX allows running a transfer path analysis in any case such as a quick troubleshooting measurement to identify a low-parameterized model based on a small amount of measurement data (e.g. only targets and no additional indicators) or a more refined and accurate analysis using a more complex model together with additional measurements (targets and extra indicators).

\subsection{Quality indicators}

However, this strength can become a weakness if the engineer has no clue on which amount of measurement data (e.g. number of response points, single or multiple orders, etc.) and what model complexity (e.g. width of frequency bands, impose parameter relations, etc.) to use. In this respect the aforementioned OPAX considerations are an essential part of the method. Furthermore, the use of quality indicators guiding the engineer through an OPAX exercise has the same importance.

Like in every LS parameter estimation problem, the (i) condition number and the (ii) model fit or synthesis of the output data are good candidates for quality indicators. An OPAX analysis can be considered reliable if the condition number is low and the model synthesis is good.

In case the condition number is high, the OPAX model complexity is probably too high for the dynamics present in the measurement data and will lead to unreliable parameter estimates with large standard deviations. In such case, one has two options. The first one is to simplify the model, reducing the number of parameters. This can be done, for example, using a priori information of the mounts when available, or taking a multi-band model with larger frequency bands (one single band encompassing the complete frequency range under study) and accept reduced model accuracy. The second option is to use more orders and/or to include extra indicators at the cost of increased FRF measurement efforts.

Looking at the quality of the synthesis, it can be concluded that a poor fit of the response data means a low model quality. This can be due to errors in the measurement data (e.g. noisy data, shifted frequency peaks in the FRF data, etc.) but also because of using an overly simplified model. To improve the results the measurement data needs to be checked for consistency and more complex models, adequately describing the system behavior, need to be used.

It should be noted that the opposite is not necessarily true, a good synthesis does not always correspond to good model quality. In particular, in case the target response is already used for the model parameter estimation, the same data are used to derive the model and to validate the response synthesis. Also, in this case the method can fail to indicate neglected paths as their effect is implicitly taken up in the considered paths. For these reasons it is a good practice to use the indicators in the model estimation and the targets in the synthesis step, or at least do the synthesis validation with targets that were not included in the modeling step. This way the backward-forward use of the same data is avoided.

\section{Example}

A simulation study was carried out to investigate and validate the OPAX method. The basis of the simulations was an engine TPA dataset of a 4-cylinder car in run-up condition (1000-6000 RPM) from which a subset of 18 structural paths was selected (4 engine mounts and 2 subframe mounts in 3 directions), as depicted in Fig. 6 . This subset contained 1 pressure target and 21 acceleration indicators. Acoustic contributions were not considered in this study. The following measured data was available: (i) 18 operational active and passive side mount accelerations, all described in terms of orders from 0.5 up to 10 ; (ii) 18 transfer path FRFs to the pressure target; and (iii) a $21 \times 18$ body FRF matrix between all forces and indicator points. The dataset was extended to be suitable for an OPAX analysis by adding 18 mount stiffness profiles, each having SDOF characteristics. The simulated operational forces were calculated from the active and passive side accelerations and the mount stiffness profiles, and finally, the target and indicator responses were simulated using these forces and the available FRFs.

Due to this bottom-up approach of building the simulation dataset, the results of the OPAX analysis agreed perfectly with the simulation. To test the method in a more real-life scenario, the operational mount accelerations and FRFs were contaminated with noise, then the target and indicator responses were resynthesized using this noisy dataset.

First, an OPAX analysis was carried out using an SDOF model. The SDOF model was identified based on a limited subset of only 3 indicators and using all 20 order components. As an example, Fig. 7 shows the obtained order 2 path contributions in comparison with those of the true reference TPA model. Fig. 8 compares the estimated dynamic mount stiffness characteristics with the true SDOF profiles for one of the mounts in the axial $z$-direction. The critical paths are clearly well captured and the mount stiffness estimates are very good as well.

In a second analysis, a multi-band model was used. A large number of scenarios were tested using various combinations of (i) frequency bandwidth $(5,10, \ldots, 120 \mathrm{~Hz})$, (ii) number of indicators $(1,2, \ldots, 21$ indicators) and (iii) number of orders ( $1,2, \ldots, 20$ orders). The dynamic mount stiffness profiles and path contributions were identified for all these contributions. The condition number was calculated as quality indicator and the error of the mount stiffness estimations was quantified by the normalized least squares (NLS, in \%) error. 


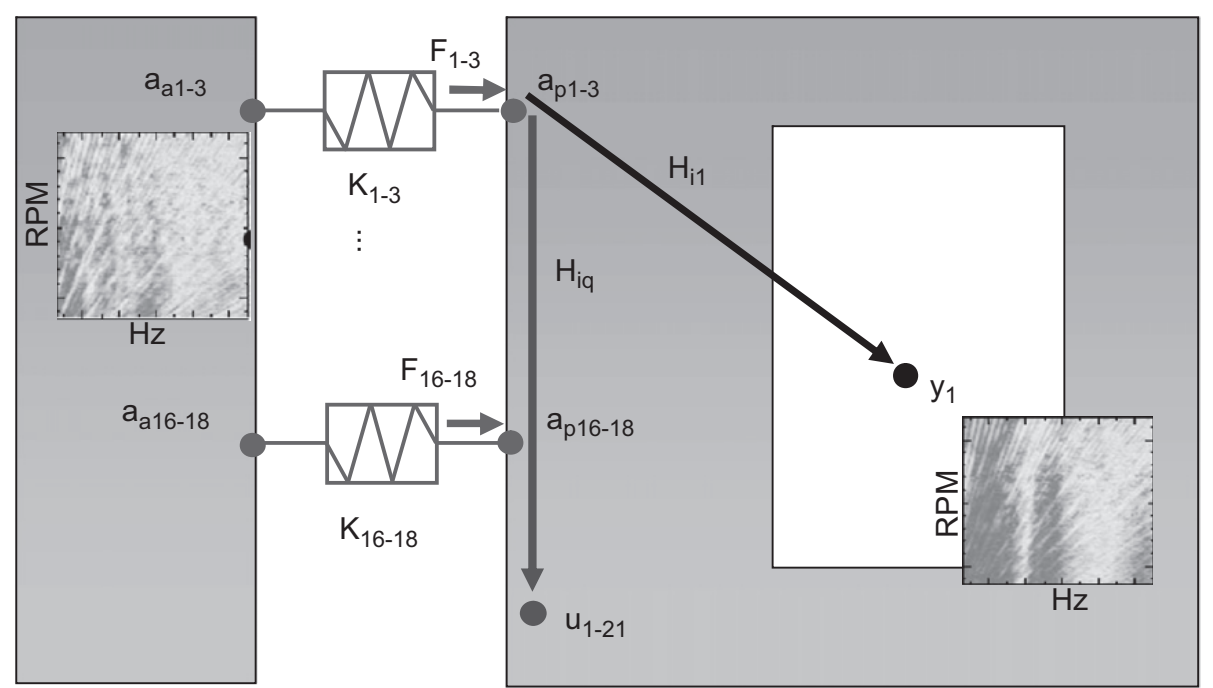

Fig. 6. OPAX example with 18 structural paths ( 6 mounts in 3 directions), 1 pressure target and 21 extra acceleration indicators.

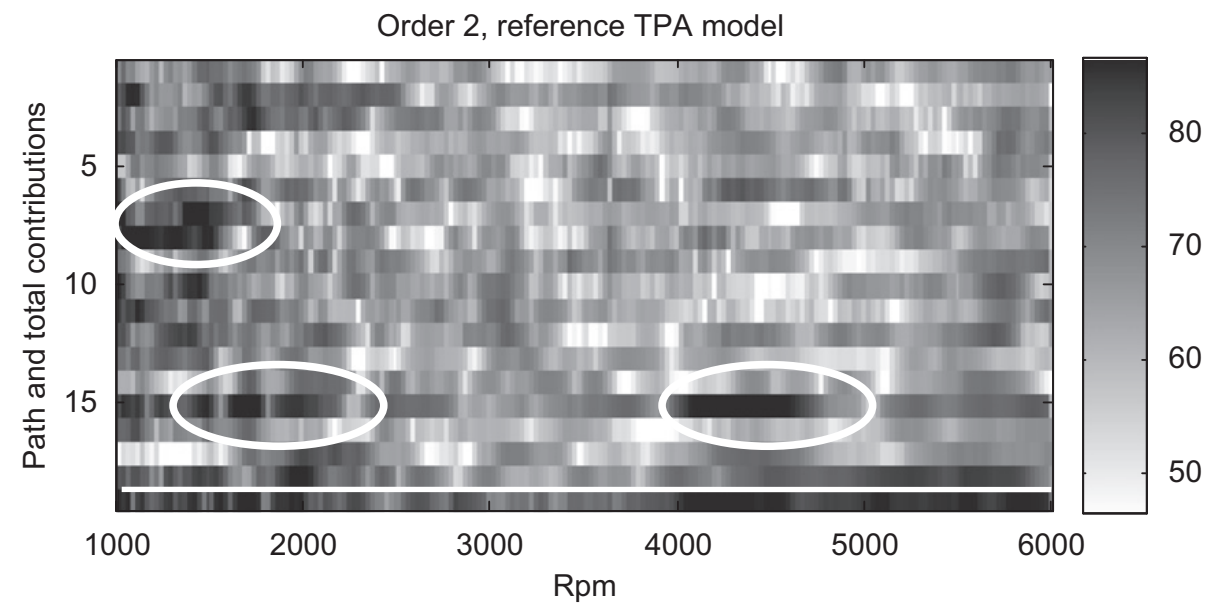

Order 2, OPAX SDOF using 3 indicators

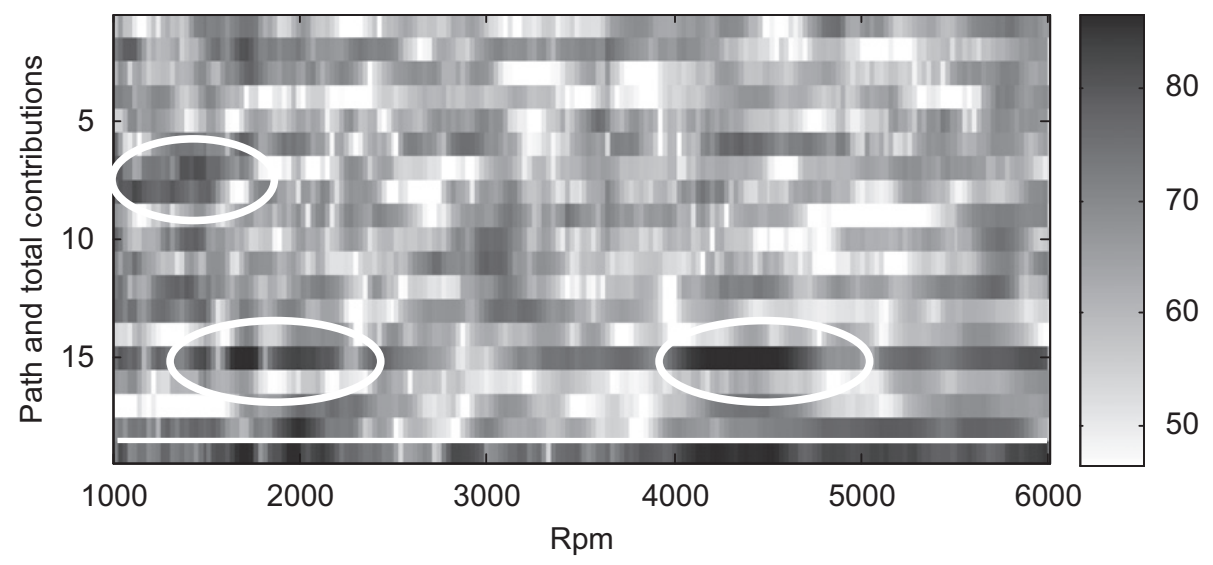

Fig. 7. OPAX SDOF order 2 path contributions in comparison with the reference TPA model.

Some of the results are presented in Figs. 9 and 10, showing the condition number (graph a) and NLS error of the mount stiffness estimations (graph $b$ ) in function of the used number of orders ( $x$-axis) and indicators ( $y$-axis) for two different cases with a small $(5 \mathrm{~Hz})$ and a larger $(55 \mathrm{~Hz})$ bandwidth. The following observations can be made:

- First of all, there is clearly a good agreement between the condition number and the mount stiffness estimation error. The parametric estimations improve with decreasing condition number. This clearly confirms the condition number as a good quality indicator as proposed in the previous section. 

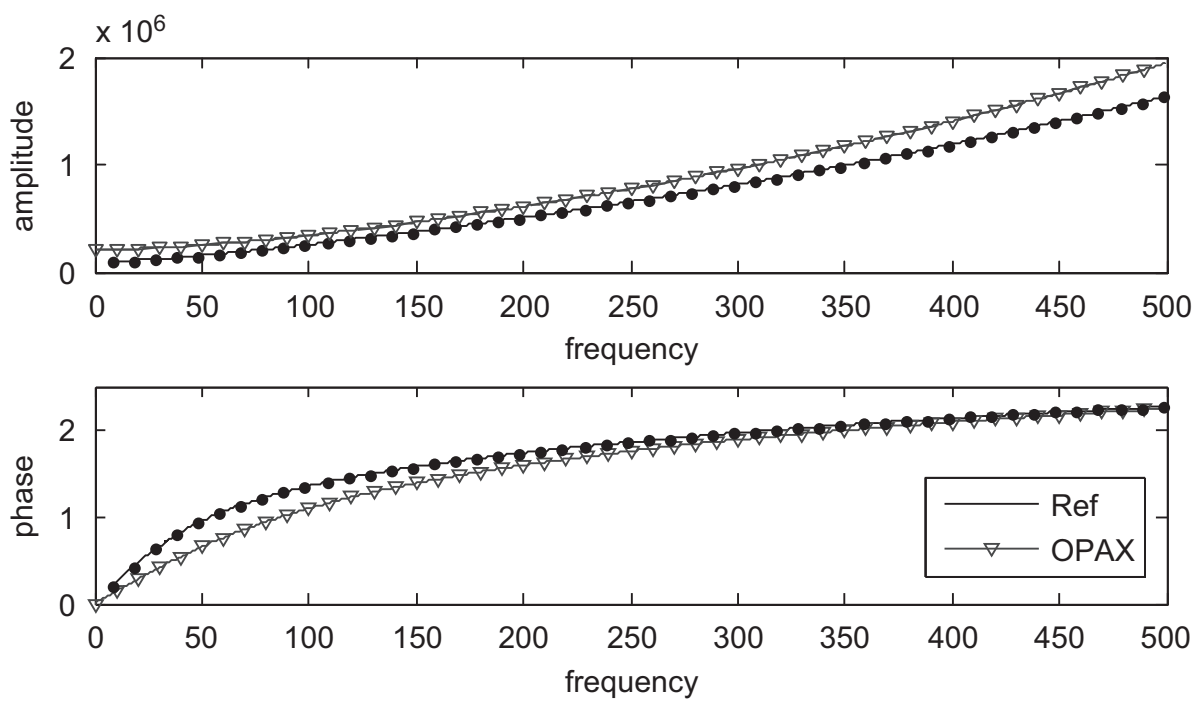

Fig. 8. Comparison of estimated mount stiffness characteristics with the true SDOF profile for one of the mounts (mount 2 in axial $z$-direction).

a

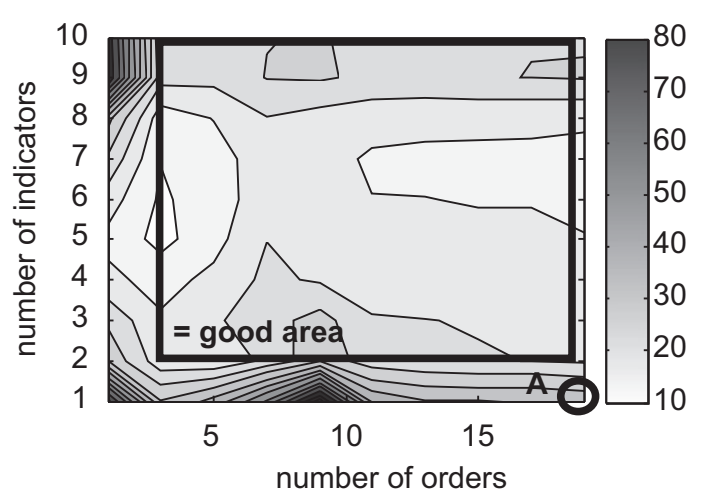

C

OPAX results for point $A$ : stiffness estimates for mount 2 in z-direction
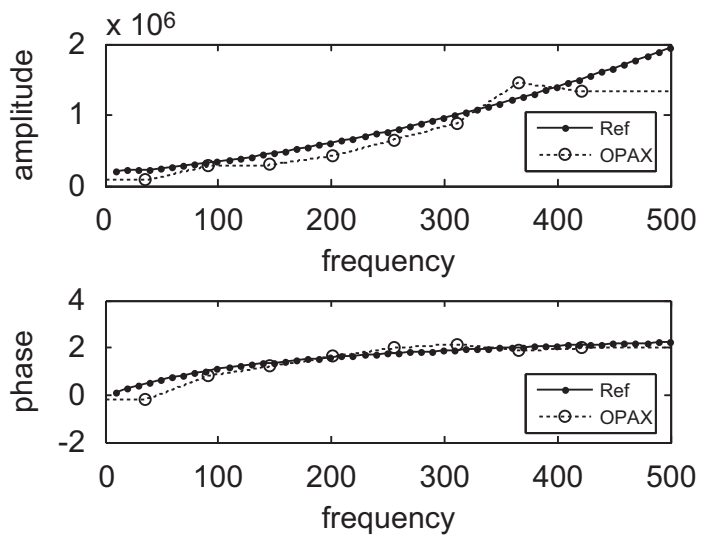

b

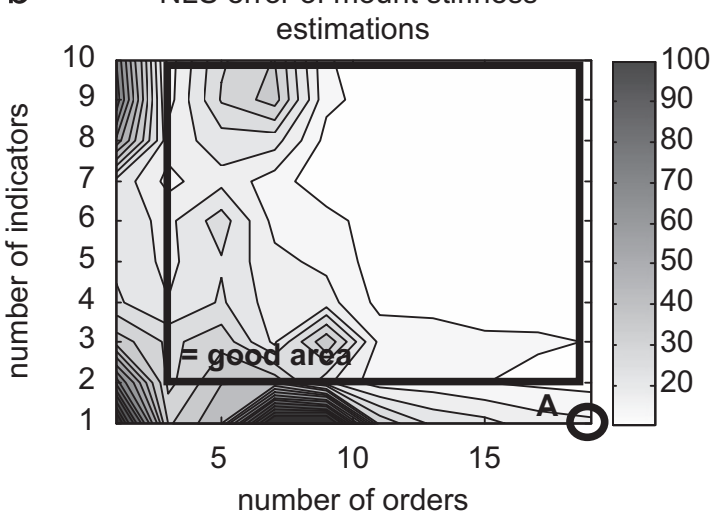

d

OPAX results for point $A$ : order 2 fit of indicator 1

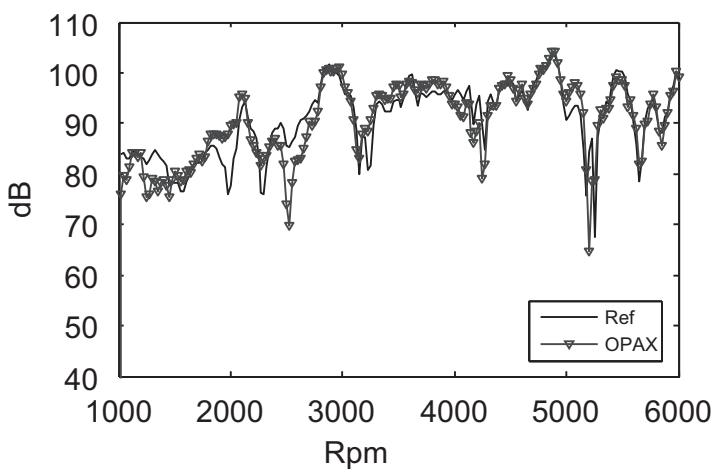

Fig. 9. OPAX multi-band analysis using bands of $55 \mathrm{~Hz}$ : (a) condition number and (b) NLS error (in \%) of the mount stiffness estimations in function of the used number of orders ( $x$-axis) and indicators ( $y$-axis); (c) mount stiffness estimates and (d) order 2 synthesis results for OPAX scenario A (1 indicator, 20 orders).

- Next to this, it is interesting to see the differences between the 5 and $55 \mathrm{~Hz}$ analysis cases:

$\circ$ When applying frequency bands of $55 \mathrm{~Hz}$, most of the investigated scenarios give good results as shown in Fig. 9(a) and (b). There is a large blue area on the contour plots with a low condition number (less than 30) and small mount stiffness estimation errors (NLS less than 20\%). If multiple orders are used, good results can already be obtained when measuring only one response point. Fig. 9(c) and (d) illustrates this, for example, for point A (1 indicator, 20 orders). The mount stiffness estimations and the total OPAX synthesis match the original data well. This example is a good illustration that 
a

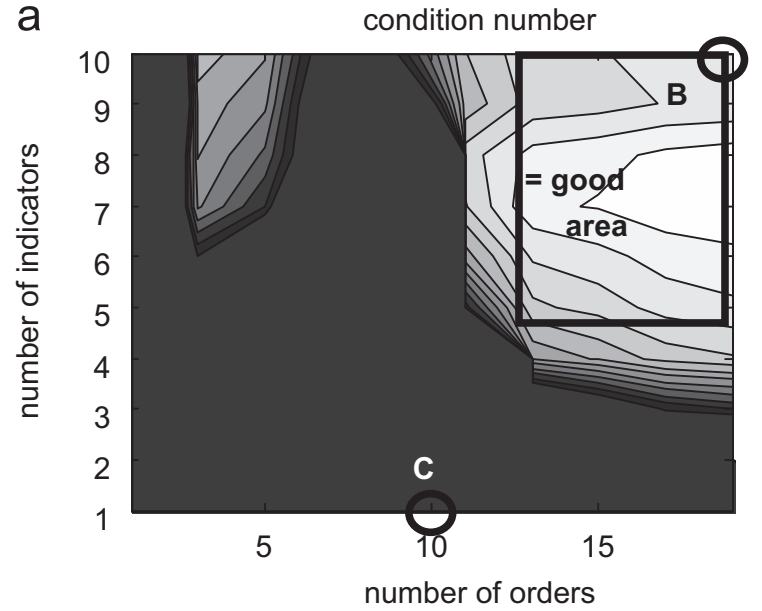

C

OPAX results for point $B$ :

stiffness estimates for mount 2 in z-direction
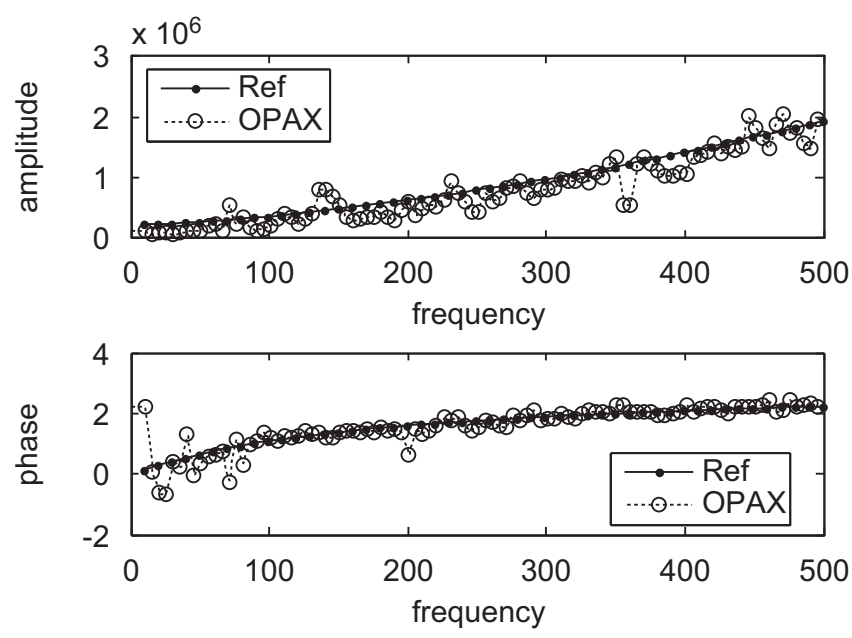

e

OPAX results for point $\mathrm{C}$ :

stiffness estimates for mount 2 in z-direction
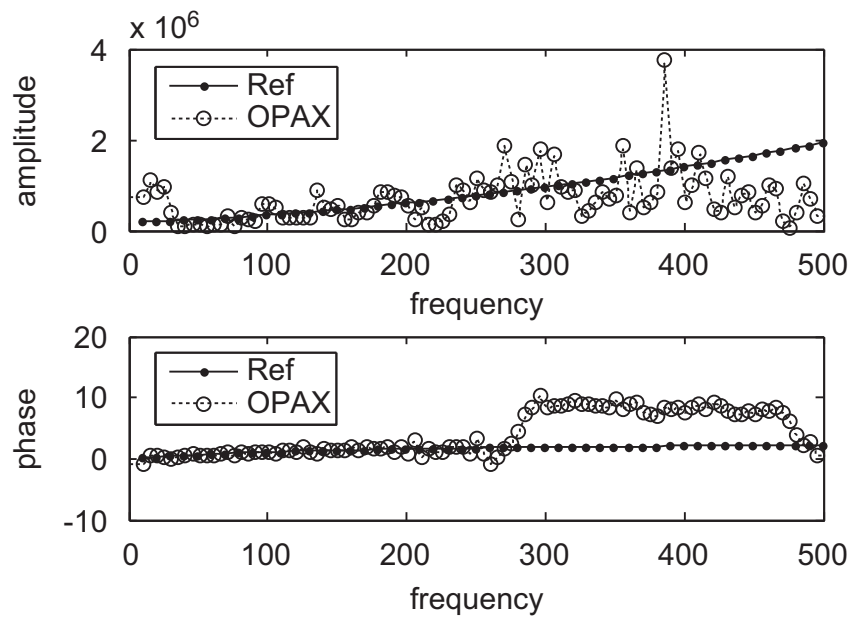

b

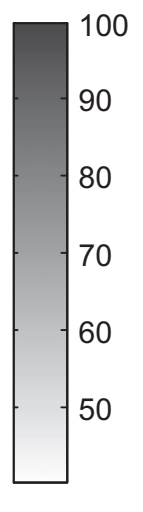

d

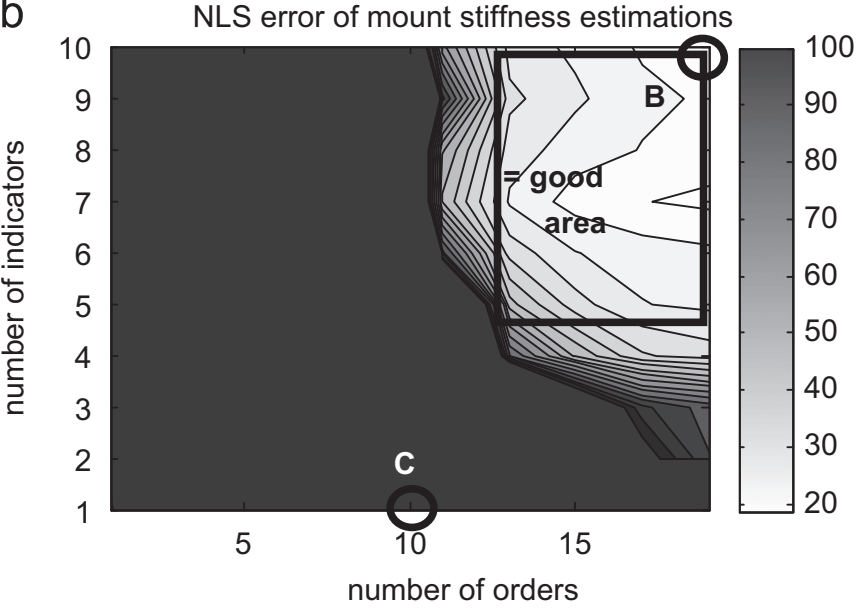

OPAX results for point $B$ : order 2 fit of indicator 1

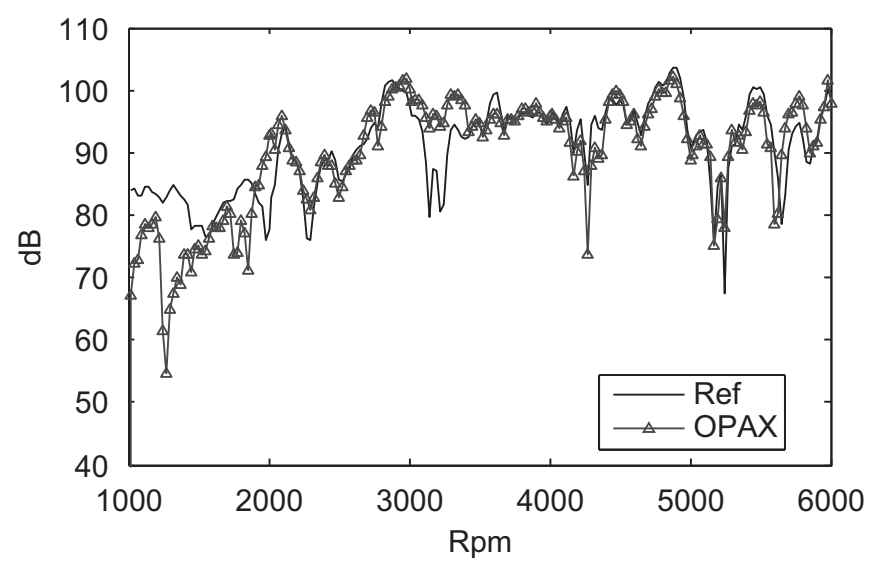

f

OPAX results for point $\mathrm{C}$ : order 2 fit of indicator 1

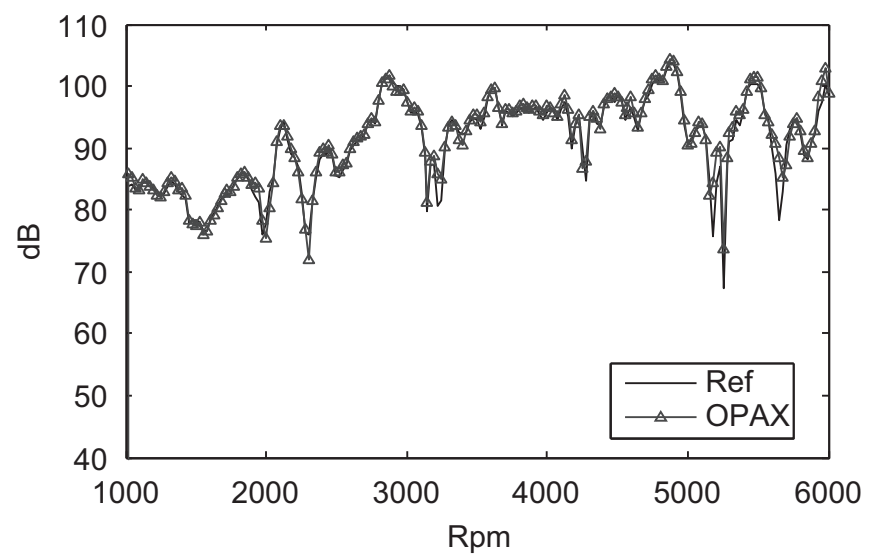

Fig. 10. OPAX multi-band analysis using small bands of $5 \mathrm{~Hz}$ : (a) condition number and (b) NLS error (in \%) of the mount stiffness estimations in function of the used number of orders ( $x$-axis) and indicators ( $y$-axis); (c,e) mount stiffness estimates and (d,f) order 2 synthesis results for OPAX scenario's B (10 indicators, 20 orders) and C (1 indicator, 10 orders).

in such case there is no need to measure more than one response point. This is an incredible gain compared to matrix inversion TPA where about 36 response points should be measured in order to identify the loads.

$\circ$ When using small bands of $5 \mathrm{~Hz}$, only a few investigated scenarios gave good results as shown in Fig. 10(a) and (b). The blue area in the contour plots with low condition number (less than 30) and small mount stiffness estimation error 
(NLS error less than 20\%) is much smaller than in the first case with $55 \mathrm{~Hz}$ bands. Good results are only obtained when using a high number of orders and indicators. Fig. 10(c) and (d) illustrates this, for example, for point B in the contour plots (10 indicators, 20 orders). The OPAX results become worse when taking less indicators and orders. This is shown in Fig. 10(e) and (f) for point C (1 indicator, 10 orders). The reason for this is that a high number of model parameters need to be estimated from insufficient measurement data. This inevitably leads to spiky, unreliable mount stiffness estimates and finally also to incorrect path contributions. The total OPAX synthesis on the other hand is excellent, showing almost no difference compared to the measurement data. This can be explained by the fact that the OPAX model is far over-parameterized, which results in an excellent fit but poor parameter estimation. So, in this example with limited measurement data, taking larger bands (e.g. $55 \mathrm{~Hz}$ as in case 1) is the only way to obtain reliable results with good parameter estimations.

Looking at the contour plots for all investigated frequency bandwidths $(5,10, \ldots, 120 \mathrm{~Hz})$, the following rule of thumb can be formulated for OPAX, combining the different requirements to obtain a well-conditioned system of equations:

$$
v \operatorname{ceil}(m / 2) \operatorname{ceil}(b w / 10) \geq 2 n
$$

where $v$ is the number of indicators, $m$ is the number of orders, $b w$ is the width of the frequency bands in $\mathrm{Hz}$ and $n$ is the number of paths. This rule of thumb states that the number of indicators times half the number of orders times the frequency bandwidth in tens of $\mathrm{Hz}$ must be equal or exceed twice the number of paths. This condition is fulfilled for points $\mathrm{A}(v=1$ indicator, $m=20$ order components, $b w=55 \mathrm{~Hz}, n=18$ paths, left term $=60$, right term $=36)$ and $\mathrm{B}(v=10$ indicators, $m=20$ order components, $b w=5 \mathrm{~Hz}$, $n=18$ paths, left term $=100$, right term =36) explaining the good results in Figs. 9(c) and (d) and 10(c) and (d). It is not fulfilled for point $C(v=1$ indicator, $m=10$ order components, $b w=5 \mathrm{~Hz}, n=18$ paths, left term=5, right term=36), where the amount of measurement data per frequency band is far too low to properly estimate the mount stiffness parameters.

This OPAX rule of thumb also holds for classical TPA matrix inversion, which is actually a very specific case of OPAX ( $m=1$ order, $b w=1 \mathrm{~Hz})$ as mentioned before. There, we need at least twice as many indicators as paths $(v \geq 2 n)$, confirming the classical TPA rule of thumb [25,12].

\section{Conclusions}

A new TPA method was developed, combining the speed of the operational path method (OPA) and the effectiveness of the traditional TPA methods. The new method is referred to as operational path analysis with eXogeneous inputs (OPAX). The key feature is the use of parametric load models characterizing the operational forces and acoustic loads in function of measured path inputs such as mount accelerations and pressures. The parametric load models are estimated from (i) in-situ measured operational data (path inputs, target(s) and optionally additional indicators) and (ii) FRF measurement data. The OPAX method has several advantages:

- The strength of OPAX lies in the use of low-parameterized models, requiring the estimation of only a limited number of model parameters. Next to this, multiple frequencies (complete frequency band in case of SDOF model and all frequencies per band in the multi-band model) and order components (up to the user to include one, multiple or all orders) can be included simultaneously in the system of equations, drastically reducing the number of response points needed to properly identify the model. This overcomes the need to measure a large matrix of FRFs due to a high number of indicator points and thus strongly reduces measurement time and effort.

- OPAX is a scalable method, enabling the engineer to use a simple model based on a small amount of measurement data for quick troubleshooting or increase accuracy using a more complex model together with additional measurements.

- The measurement efforts are small compared to the traditional matrix inversion TPA method. Next to the operational measurements of path inputs and target(s), OPAX requires in many cases only one reciprocal FRF measurement per target point, making it fast and suitable for a quick NVH troubleshooting. Of course, one can always include additional indicator points in the system of equations to increase accuracy and robustness, but still then the FRF measurement efforts remain limited compared to the traditional TPA matrix inversion method, where the loads are identified on a frequency-by-frequency and order-by-order basis and the system of equations only contains one equation per indicator point.

- OPAX does not require mount stiffness data. Such data is often not available and not always accurate, which is a major bottleneck for the traditional mount stiffness TPA method.

- The estimated model parameters allow determining additional interesting information of the system. For example, the ability of estimating the dynamic mount stiffness characteristics from OPAX measurement data is an interesting additional feature of the method.

- The estimation of the parametric load models is numerically stable. Ill-conditioning problems resulting from pseudoinversion of rank-deficient datasets like those in operational path analysis (OTPA) hardly occur.

- The bandwise estimation in OPAX is less sensitive to systematic FRF errors (e.g. shifts of frequency peaks) than the classical frequency-by-frequency matrix inversion technique.

- OPAX allows a simultaneous identification of structural and acoustic loads and paths. 


\section{Acknowledgements}

Peter Gajdatsy, first co-author of this paper, is an early stage researcher at LMS International and K.U. Leuven under the Smart Structures Marie Curie Research Training Network (MRTN-CT-2006-035559). The financial support of the European Commission is gratefully acknowledged.

\section{References}

[1] J.S. Bendat, A.G. Piersol, Engineering Applications of Correlation and Spectral Analysis, Wiley, New York, 1980.

[2] F.X. Magrans, Method of measuring transmission paths, J. Sound Vib. 73 (3) (1981) 321-330.

[3] J. Verheij, Multipath sound transfer from resiliently mounted shipboard machinery. Ph.D. Dissertation, Technische Physische Dienst TNO-TH, Delft, 1986.

[4] D. de Vis, W. Hendricx, P. van der Linden, Development and integration of an advanced unified approach to structure boom noise analysis, in: Proceedings of the 2nd International Conference on Vehicle Comfort, ATA, 1992.

[5] P. van der Linden, T. Keppens, J. Raff, J. Schnur, Determination of the noise contributions of engine surfaces, in: Proceedings of the SAE Noise and Vibration Conference, US, 2001, Paper No. 2001-01-1482.

[6] P. van der Linden, K. De Meester, Airborne source strength measurements on the basis of pressure indicator inversion applied to an engine valve cover in: Proceedings of the 4th ICSV, St-Petersburg, 1996.

[7] K. Wyckaert, F. Augusztinovicz, P. Sas, Vibro-acoustical modal analysis: reciprocity, model symmetry, and model validity, J. Acoust. Soc. Am. 100 (5) (1996) 3172-3181.

[8] F.J. Fahy, Vibro-acoustic reciprocity principle and applications to noise control, Acustica 81 (6) (1995) 544-558.

[9] J. Verheij, Experimental procedures for quantifying sound paths to the interior of road vehicles, in: Proceedings of the 2nd International Conference on Vehicle Comfort, Bologna, Italy, October 14-16, 1992, pp. 483-491.

[10] J. Starkey, G. Merril, On the ill-conditioned nature of indirect force measurement techniques, Int. J. Analyt. Experim. Modal Anal. 4 (3) (1989) 103-108.

[11] R. Freymann, R. Stryczek, Noise path analysis and optimization, in: Proceedings of the Unikeller Conference'95, Zurich, May $30-J u n e ~ 1,1995$.

[12] P. van der Linden, H. Floetke, Comparing inverse force identification and mount stiffness force identification methods for noise contribution analysis, in: Proceedings of the ISMA 2004, Leuven, Belgium, September 2004.

[13] P. Mas, P. Sas, K. Wyckaert, Indirect force identification based upon impedance matrix inversion: a study on statistical and deterministical accuracy, in: Proceedings of the ISMA 19, Leuven, Belgium, September 1994.

[14] H. Van der Auweraer, P. Mas, S. Dom, A. Vecchio, K. Janssens, P. Van de Ponseele, Transfer path analysis in the critical path of vehicle refinement: the role of fast, hybrid and operational path analysis, in: Proceedings of the SAE Noise and Vibration Conference, May 14-17, 2007, Paper No. 2007-01-2352.

[15] W. Biermayer, F. Brandl, R. Hoeldrich, A. Sontacci, S. Brandl, W. Fliesser, Sound engineering based on source contributions and transfer path results, in: Proceedings of the JSAE 2007, 20075399, Yokohama, Japan, May, 2007.

[16] K. Noumura, J. Yoshida, Method of transfer path analysis for vehicle interior sound with no excitation experiment, in: Proceedings of the FISITA 2006, Yokohama, Japan, October 2006, Paper No. F2006D183.

[17] D. de Klerk, M. Lohrmann, M. Quickert, W. Foken, Application of operational transfer path analysis on a classic car, in: NAG/DAGA 2009 International Conference on Acoustics, Rotterdam, 23-26 March 2009.

[18] N.M.M. Maia, J.M.M. Silva, A.M.R. Riberio, The transmissibility concept in multi-degree-of-freedom systems, Mechan. Syst. Signal Process. 15 (1) (2001) 129-137, doi:10.1006/mssp.2000.1356 ISSN 0888-3270.

[19] P. Gajdatsy, K. Janssens, W. Desmet, H. Van der Auweraer, Application of the transmissibility concept in transfer path analysis, Mechan. Syst. Signal Process. 24 (7) (2010) 1963-1976, doi:10.1016/j.ymssp.2010.05.008 Special issue: ISMA 2010.

[20] C. Devriendt, P. Guillaume, Identification of modal parameters from transmissibility measurements, J. Sound Vibr. 314 (1-2) (2008) $343-356$.

[21] K. Janssens, P. Gajdatsy, H. Van der Auweraer, Operational path analysis: a critical review, in: Proceedings of the ISMA 2008, Leuven, Belgium, September 2008.

[22] W. Biermayer, F. Brandl, R. Hoeldrich, A. Sontacci, S. Brandl, H.H. Piebsch, Efficient transfer path analysis for vehicle sound engineering, in: Proceedings of the of JSAE 2008, Yokohama, Japan, May, 2008, Paper No. 20085030.

[23] D. de Klerk, A. Ossipov, Operational transfer path analysis: theory, guidelines \& tire noise application, MSSP 24 (7) (2010) $1950-1962$.

[24] H. Van der Auweraer, L. Hermans, Multivariate correlation analysis of non-stationary signals: application to pass-by noise problems, in: Proceedings of the IEEE-ICASSP 2000, vol. VI, Istanbul, June 5-9 2000, pp. 3903-3906.

[25] W. Hendrickx, Accurate vehicle FRF measurements for indirect force determination based upon matrix inversion, in: Proceedings of the ISMA 19, Leuven, Belgium, 1994

[26] C. Papadimitriou, Optimal sensor placement methodology for parametric identification of structural systems, J. Sound Vibr. 278 (4-5) (2004) 923-947, doi:10.1016/j.jsv.2003.10.063 ISSN 0022-460X.

[27] K.V. Yuen, L.S. Katafygiotis, C. Papadimitriou, N.C. Mickleborough, Optimal sensor placement methodology for identification with unmeasured excitation, J. Dyn. Syst. Meas. Contr. 123 (4) (2001) 677-686.

[28] I. Bruant, L. Gallimard, S. Nikoukar, Optimal piezoelectric actuator and sensor location for active vibration control, using genetic algorithm, J. Sound Vibr. 329 (10) (2010) 1615-1635, doi:10.1016/j.jsv.2009.12.001 ISSN 0022-460X

[29] N.J. Kessissoglou, P. Ragnarsson, A. Lofgren, An analytical and experimental comparison of optimal actuator and error sensor location for vibration attenuation, J. Sound Vibr. 260 (4) (2003) 671-691, doi:10.1016/S0022-460X(02)00987-2 ISSN 0022-460X.

[30] M. Blau, Force spectra identification by FRF matrix inversion: a sensor placement criterion, J. Acoust. Soc. Am. 105 (2) (1999) 970.

[31] A.N. Thite, D.J. Thompson, Selection of response measurement locations to improve inverse force determination, Appl. Acoust. 67 (8) (2006) 797-818.

[32] G. Desanghere, Source identification methods based on input/output transferfunction measurements: coherence analysis and energy allocation, in: Proceedings of the ISMA 8, Leuven, Belgium, 1983.

[33] D. de Klerk, D.J. Rixen, Component transfer path analysis method with compensation for test bench dynamics, Mechan. Syst. Signal Process. 24 (6) (2010) 1693-1710. 